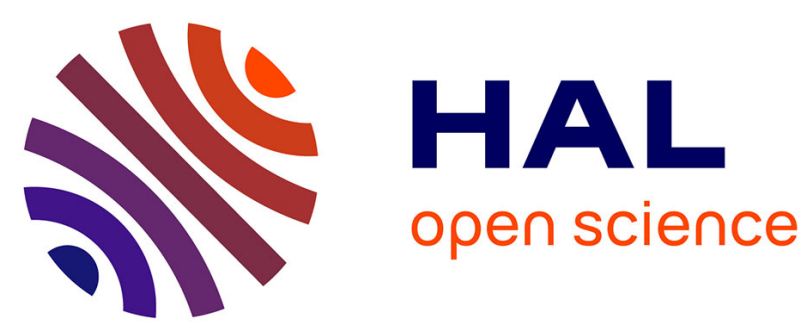

\title{
Priam ou la force de l'âge
}

David-Artur Daix

\section{To cite this version:}

David-Artur Daix. Priam ou la force de l'âge. Mètis - Anthropologie des mondes grecs anciens, 2009, 7, pp.137-170. 10.4000/books.editionsehess.2467 . hal-00697829

\section{HAL Id: hal-00697829 https://hal.science/hal-00697829}

Submitted on 4 Mar 2020

HAL is a multi-disciplinary open access archive for the deposit and dissemination of scientific research documents, whether they are published or not. The documents may come from teaching and research institutions in France or abroad, or from public or private research centers.
L'archive ouverte pluridisciplinaire HAL, est destinée au dépôt et à la diffusion de documents scientifiques de niveau recherche, publiés ou non, émanant des établissements d'enseignement et de recherche français ou étrangers, des laboratoires publics ou privés. 


\author{
DAVID-ARTUR DAIX \\ École Normale Supérieure, Paris
}

\title{
PRIAM OU LA FORCE DE L' ÂGE ${ }^{1}$
}

Au début du Chant III de l'Iliade, Hector propose à son frère Pâris de décider par un duel avec Ménélas l'issue de la guerre de Troie. Le défi est lancé et l'Atride en accepte le principe, tout en posant ses conditions. En effet, il ne s'en remettra pas aux fils de Priam pour régler le combat, tant il se défie d'eux, mais au vieux roi en personne :

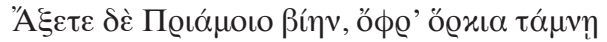

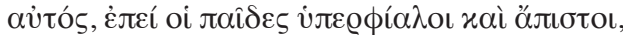

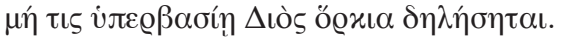

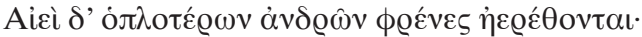

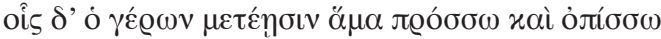

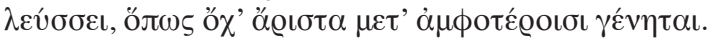

Amenez ici Sa Force Priam, afin qu'il conclue le pacte en personne, puisque ses fils sont arrogants et déloyaux.

Il ne faut pas que quelqu'un, par une extravagance, fasse tort au pacte de Zeus.

L'esprit des jeunes hommes toujours flotte à tout vent.

Quand un vieillard est avec eux, en rapprochant l'avenir du passé,

il voit comment il est possible d'arranger tout au mieux pour les deux parties.

(III.105-10)

Les raisons que Ménélas invoque, fondées sur l'opposition entre jeunesse et vieillesse, sont traditionnelles. Les jeunes gens jouissent de la vigueur. Désignés ici par l'épithète ó $\pi \lambda$ ó $\tau \varepsilon \varrho o l-c o m p a r a t i f$ formé sur $\tau \grave{\alpha}$ ǒ $\pi \lambda \alpha$ : les «armes »- , leur domaine est la guerre et l'épreuve de force, mais non le conseil. Les vieillards, au rebours, sont faibles, assurément, mais brillent par leur sagesse. Au moment de conclure un pacte, la vieillesse de

1. Cet article est dédié à la mémoire de Jean-Pierre Vernant et Pierre Vidal-Naquet. 
l'arbitre garantit un jugement équitable. Les jeunes princes, en revanche,

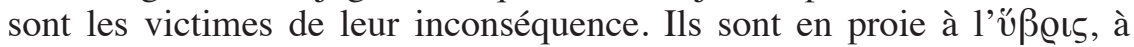
un comportement excessif ( $\dot{\tau} \pi \varepsilon \varrho \phi i ́ \alpha \lambda \mathrm{ol}$ ), prêts à toutes les transgressions

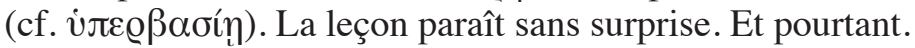

Chaque mot a ici son importance. Ménélas, quand il réclame Priam, n'évoque pas sa sagesse, au contraire. Dans son édition, Paul Mazon traduit par «le puissant Priam $»^{2} l$ 'expression que nous rendons ici par « Sa Force

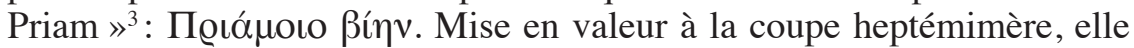
signifie littéralement « la force de Priam » et sonne très étrangement pour désigner le souverain troyen. En effet, si, en français, on peut comprendre qu'il est fait mention ici de l'autorité du roi, de sa « puissance », en réalité, la «force» dont il est question ici, la ßín, est en grec toute physique, souvent martiale, violente et brutale. Il y a donc là une incongruité dans le choix de cette formule qu'emploie Ménélas - c'est presque un oxymore - qui mérite qu'on s'y arrête.

\section{La force de la jeunesse}

Entre tous les termes qui désignent la vigueur, ßí $\eta$ et - d'un emploi plus rare $-i \varsigma^{4}$, que l'on retrouve dans la vis latine, sont ceux qui renvoient le plus à une qualité proprement physique et innée du héros ${ }^{5}$. En grec, cependant, seule la ßín comprend régulièrement l'idée de violence, de contrainte, d'excès brutal ${ }^{6}$. Elle couvre un domaine plus vaste que ses synonymes, rend compte de la vigueur sous toutes ses facettes, tantôt bonnes, tantôt funestes. En ce sens, elle est « primordiale ».

2. Les traductions que je propose dans cet article s'inspirent largement de celle de Paul Mazon, même si, afin de mieux suivre la progression des vers et dans un souci de précision lexicale et syntaxique, il m'arrive parfois de la modifier légèrement. Les Chants de l'Iliade sont numérotés en chiffres romains, ceux de l'Odyssée en chiffres arabes.

3. J'emprunte cette traduction à Eugène Lasserre (L'Iliade, Paris, 1965), qui imite probablement ici Victor Bérard dans son édition de l'Odyssée (cf. «Sa Force et Sainteté Télémaque »). Robert Flacelière traduit quant à lui, pour la Bibliothèque de la Pléiade, « le puissant roi Priam » (Iliade-Odyssée, Paris, 1955).

4. Sur la synonymie entre ces deux termes, cf. Gregory NAGY, The Best of the Acheans : Concepts of the Hero in Greek Archic Poetry, Baltimore et Londres, $2^{\mathrm{e}}$ édition 1999, chapitre V, $\S 37$.

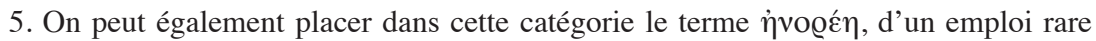
(cinq occurrences seulement dans l'Iliade, une dans l'Odyssée). Formé sur le mot àví@,

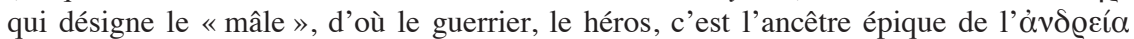
classique: le courage viril.

6. Cf. Pierre Chantraine, Dictionnaire étymologique de la langue grecque, s.v. ßía, p. 174 . 


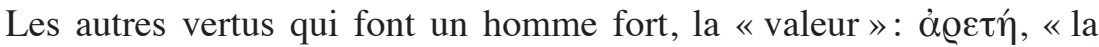

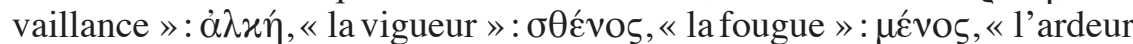

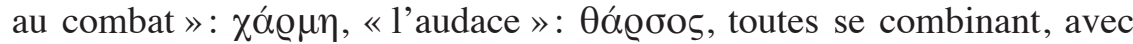
d'autres éléments encore, dans le x@ó un prêt plutôt qu'un véritable don. Les dieux « insufflent » cette vigueur-là aux mortels, mais seulement le temps d'un duel ou d'une bataille. Athéna favorise de la sorte Diomède au début de ce qui sera son aristie (V.1-3 ; voir aussi V.125). Il en va de même au cours de la « Dolonie » (cf. X.274 $s q$. , en particulier les vers 366 et 482) où Diomède est accompagné d'un autre favori de la déesse, Ulysse; ou lors de la fameuse course de char au Chant XXIII (cf. 388 sq.) ${ }^{7}$; et dans bien d'autres circonstances encore ${ }^{8}$. Comme Énée le rappelle à Achille au moment de l'affronter, «Zeus

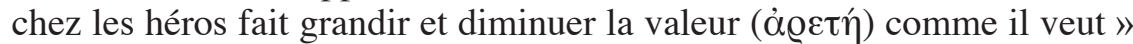
(XX.242-3 ; voir aussi XV.93). De leur côté, les héros s'efforcent de faire montre de ces qualités. Mais, précisément, elles ne vont pas de soi.

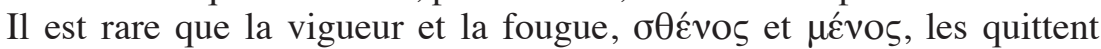
entièrement: sans doute s'apparentent-elles le plus à la ßí Avec le mot $\mu$ ćvo sependant, la vigueur du héros glisse de ses membres vers son cœur, du domaine purement physique vers celui des sentiments ${ }^{10}$, moins constant sans doute, plus sujet aux variations. Preuve que le $\mu$ ćvo ne vaut pas tout à fait la ßín. Hector, au moment d'affronter Achille au Chant XXII, s'imagine que le Péléide veut lui faire peur pour qu'il en oublie

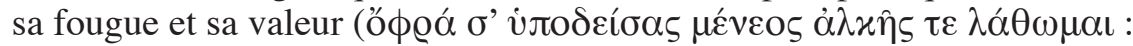
XXII.282). Toutefois, un rapprochement avec le vers $265 \mathrm{du}$ Chant vi montre que la fougue reste associée aux membres du héros. Hector demande à Hécube de ne pas lui donner de vin afin de ne pas « faire sortir la fougue de ses membres », de peur qu'il en oublie sa vaillance ( $\mu \eta \dot{~} \mu$ '

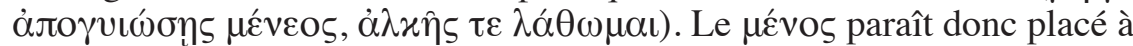
mi-chemin entre la force physique et la force morale ${ }^{11}$. Plus stable que la

7. Cf. en particulier les vers 390 et 400.

8. Les scènes où un dieu insuffle l'une ou l'autre de ces qualités aux héros (ou parfois, quand ce sont eux qui importent, à leurs coursiers) abondent: II.451; V.513; VIII.140; etc.

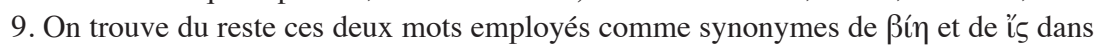
le type de formules qui nous intéresse: cf. Gregory NAGY, The Best of the Acheans cité, chapitre V, § 37 ; et infra « Une formule archaïque ».

10. Cf. XVII.20-3: les fils de Panthoos nourrissent leur $\mu$ ćvos comme on le fait d'un

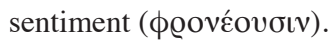

11. Voir aussi IX.705-6; 22.226. Certes, ce partage n'est pas absolu: quand la vaillance et la vigueur sont associées, elles emplissent tantôt les membres (cf. XVII.210-2), tantôt le cœur (cf. XVIII.499). Mais en réalité, il est aussi simple de considérer qu'à chaque fois, si un seul siège est mentionné, l'autre est implicitement associé à la vertu qui lui correspond. 
vaillance, il reste moins ferme que la ßín.

Car les héros risquent toujours de « laisser mollir leur vaillance ardente » (IV.234; VI.523; XII.409; XIII.116), de « l'oublier» (XI.313; XIII.269; XV.322; XVI.357 etc.) et avec elle « la joie du combat» (XVII.759). Les nefs achéennes seront perdues à moins qu'Achille ne revête sa vaillance (IX.231; XIX.36), comme on met une armure que l'on peut aussi bien ôter. Ajax, affrontant seul les bataillons troyens, doit alterner entre le «souvenir » de sa vaillance (XI.566) et le parti de la prudence (XI.568). Diomède, Ajax, Patrocle encouragent leurs compagnons, comme euxmêmes, à «se soucier» de leur vaillance (IV.418), à «s'en souvenir » (XV.734; XVI.270). Ils ont besoin de se rappeler la joie, l'ardeur du combat (IV.222; VIII.252; XIV.441 etc.). De même, Hector exhorte les Troyens ${ }^{12}$. Plus surprenant, Achille exige du Priamide le même effort de mémoire lors de leur duel: «rappelle ta valeur sous toutes ses couleurs » (XXII.268)! Pour que son triomphe le satisfasse, «c'est maintenant justement qu'il faut qu' [Hector] soit un combattant et un guerrier intrépide ». Ce qui suppose, au rebours, que le fils de Priam pourrait bien perdre ses moyens.

La vraie force, en revanche, celle du corps, celle des bras, la ßí

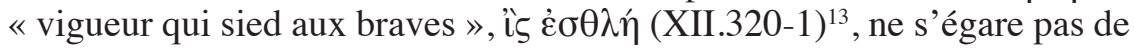
la sorte. Certes, il peut arriver qu'elle ne soit invoquée que le temps d'un combat. Au Chant VII, quand Hector et Ajax doivent s'affronter en duel, tous prient Zeus d'octroyer aux deux champions « force et gloire égales » (cf. VII.202-5). Mais, à la différence de la victoire (víxๆ) et de l'hommage

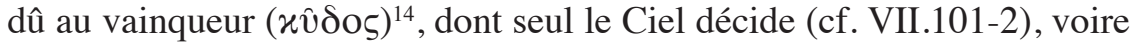
de la vaillance que Zeus contrôle à son gré15, la puissance physique, elle, n'est pas au seul pouvoir des dieux. Ajax et Hector, au moment de s'affronter, disposent déjà, et en propre, de la ßín. Elle reste un « don »du Ciel, mais un don accordé dès la naissance, au même titre que la taille ou l'intelligence (cf. VII.288-9). La vigueur du fils de Télamon est sans limite, nous dit Homère (î̀' $\dot{\alpha} \dot{c}^{\prime} \lambda \varepsilon \theta \varrho o v$ : VII.269). Quant à celle d'Hector, elle est «génétique ». Devant Andromaque, à la fin du Chant VI, il souhaitait que son fils, Astyanax, « tout jeune encore » (408) fût doté par les dieux de la

12. Cf. VI.112; VIII.174; XI.287; XV.487; XVII.185; IV.509; voir aussi XII.393; XV.380.

13. Le discours célèbre de Sarpédon (XII.310-21) dont est tirée cette formule manifeste clairement qu'à la guerre, la place des rois est aux premiers rangs et que la qualité qui les distingue alors et leur assure la gloire n'est autre que la force physique.

14. Sur le xv̂ठos, cette gloire que confère la victoire, cf. Émile BenvenISTE, Le vocabulaire des institutions indo-européennes. II : Pouvoir, droit, religion, Paris, 1969, p. 57-69.

15. Voir par exemple XVI.688-90; XVII.176-8. 


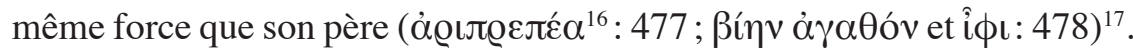
Et il en donnera la preuve là où la vigueur règne en maître : à la guerre (cf. 480-1). Les dieux interviennent bien, comme toujours, mais seulement pour permettre à la force d'Hector de s'exprimer chez Astyanax. Pour le reste, si elle est bien en lui, elle lui vient de son père.

Certes, il arrive que le héros ne soit pas assez fort par lui-même pour affronter l'ennemi.Ménélas défendant le cadavre de Patrocle ne peut contenir Hector. Athéna intervient donc et «met la force, la ßín, dans ses épaules et ses genoux »(XVII.569). Mais, en l'occurrence, il s'agit simplement d'un supplément de vigueur. Ménélas est naturellement fort. Hector l'est juste bien davantage, comme, du reste, l'avait révélé l'épisode du duel du Chant VII. Quand le fils de Priam a défié «le meilleur des Achéens qui soit » (50), Ménélas aurait bien aimé acquérir ce titre. Mais Agamemnon l'arrête: Hector est bien plus fort que lui (105) et fait peur à Achille même, qui pourtant est bien meilleur que Ménélas (114). Et c'est finalement Ajax, le tout meilleur après le Péléide $(228)^{18}$, à qui le Ciel a donné « la grandeur

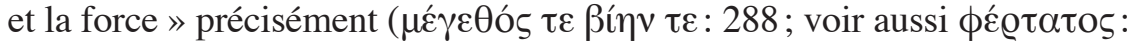
289), qui sera le champion de l'armée grecque (cf. 226-8).

Ce n'est donc pas un hasard si, dans l'Iliade, c'est Héraklès, héros fort entre tous, qui est le plus souvent défini par sa force et qui incarne le mieux

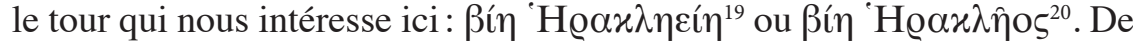
fait, cette formule est très ancienne - nous y reviendrons ${ }^{21}$ - et il semble bien que, traditionnellement, elle soit d'abord associée au fils de Zeus et d'Alcmène: Héraklès représente le « prototype » en quelque sorte.

16. Cf. Anatole BAILLY, Dictionnaire Grec-Français, Paris, 2000 (réédition), s.v. ỏ@l-, p. 265.

17. Sur la force "génétique », cf. infra, «Une formule archaïque » à propos d'Ulysse et de Télémaque dans l'Odyssée.

18. Cf. XVII.280. Sur la ressemblance entre Achille et Ajax, tous deux confiants dans leur courage viril et dans la puissance de leurs bras: cf. VIII.224-6 = XI.7-9. Achille

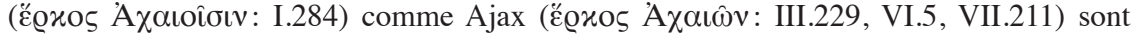
le « rempart des Achéens ». Néanmoins le fils de Télamon vient juste après le Péléide. L'Odyssée (11.550 sq.) prend sur ce point la suite de l'Iliade.

19. Cf. II.658; II.666; V.638; XI.690; XV.640; XIX.98.

20. Cf. XVIII.117; voir aussi 11.601; et dans la Théogonie d'Hésiode: is ßíns

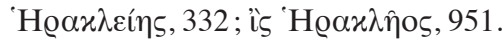

21. Cf. infra, «Une formule archaïque ». 


\section{Vieillesse ennemie}

Cependant, le fait même que la force de ces héros, leur ßín, soit « innée » constitue sa plus grande faiblesse. Elle est l'apanage de la seule jeunesse. Son pire ennemi est la vieillesse. Diomède lance ainsi à Nestor, qu'il vient de secourir sur le champ de bataille :

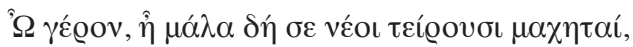

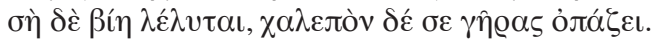

$\mathrm{Ah}$ ! vieillard, les jeunes combattants te donnent bien du mal, ta force est brisée, la fâcheuse vieillesse t'accompagne. (VIII.102-3)

La place du fils de Nélée n'est plus au combat. Il le sait bien, du reste, et passe son temps à regretter sa jeunesse et la force qui l'accompagnait:

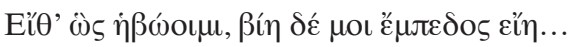

$\mathrm{Ah}$ ! si j'étais encore jeune, si ma force était intacte... (VII.157) ${ }^{22}$

Ce n'est pourtant pas le cœur qui lui manque, ni le sentiment de sa valeur passée, dont il rebat les oreilles de ses compagnons. Mais la vieillesse est un adversaire implacable. Un échange au Chant IV (310-25) entre Nestor et Agamemnon en témoigne clairement. Le vieux meneur de char ressent de manière insistante les atteintes de l'âge. Dans la bouche d'Agamemnon, l'évocation de sa force n'est plus qu'un vain souhait (314). Nestor n'en conserve que le souvenir, à l'instar de celui de ses exploits passés (318-21). Désormais, sa valeur tient dans ses conseils (323) - « c'est le privilège des vieillards » - et non dans la vigueur de ses membres. Au rebours, la force est l'essence même des jeunes guerriers :

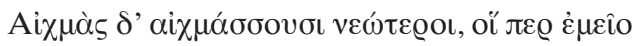

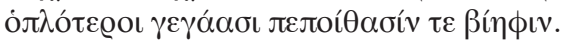

Les jeunes joueront de la lance, puisqu'ils sont plus que moi aptes à se battre et s'assurent dans leurs propres forces. (IV.324-5)

Nous retrouvons dans la conclusion de Nestor, pour désigner les jeunes gens, le même adjectif qu'employait déjà Ménélas à propos des Priamides.

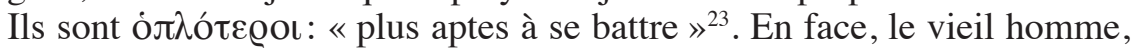

22. Cette formule revient régulièrement sur les lèvres du vieil homme: cf. IV.313 sq.; XI.668 sq.; XXIII.629 sq.

23. Chantraine note ainsi à propos de ce « comparatif différentiel », op. cit., p. 809: « il faut accepter l'explication des Anciens qui partent du sens de "capable de porter les armes, vigoureux" ". 
lui, s’il est « expert aux combats » (310), n’y a plus sa place les armes à la main, mais seulement le conseil aux lèvres ${ }^{24}$.

\section{Laforce des idées}

Reprenons l'épisode du Chant VIII où Diomède sauve Nestor, égaré sur le champ de bataille. Le fils de Tydée, comme Agamemnon plus tôt, ne voit dans la vieillesse que l'ennemie de la ßí attente, c'est finalement le fils de Nélée, tout vieux qu'il est, qui leur permet d'échapper au trépas sous les coups d'Hector. En effet, le premier, il saisit les signes envoyés par Zeus, quand Diomède, tout à sa vaillance, ne les reconnaît pas (cf. 139-40). La lucidité du vieux soldat s'avère au bout du compte aussi nécessaire que la vigueur de Diomède pour assurer le salut des deux héros.

Mais, en réalité, c'est au conseil et non à la guerre qu'un vieil homme est en droit de se faire une gloire de son âge. Ainsi, au Chant IX, alors que les Grecs discutent de ce qu'il convient de faire, maintenant que Zeus donne l'avantage aux Troyens, Nestor prend le dessus sur Diomède précisément, pour qui seule importe la vaillance, l'à $\lambda x \eta$, en lui opposant son grand âge (53-62). Il se vante ici d'être plus vieux que le fils de Tydée, dont il accentue encore la jeunesse par le superlatif d'un adjectif que nous commençons à bien connaître: Diomède, en effet, pourrait être son plus

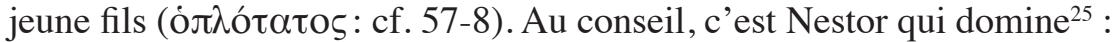

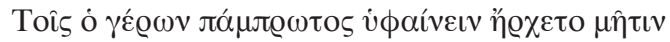

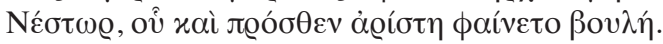

Pour eux le vieillard le tout premier commençait à ourdir les fils de son idée,

Nestor, lui dont avant aussi le conseil paraissait le meilleur. (IX.93-4)

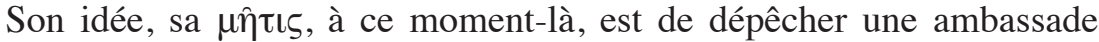
auprès d'Achille pour le convaincre de mettre un terme à sa colère. Auparavant, au Chant VII (323-5), la même formule introduisait son projet de remplacer le fils de Pélée, « le puissant rempart des Achéens contre la guerre cruelle » selon ses propres termes (I.283-4), par un mur élevé. Au

24. Nestor est très âgé et représente un cas extrême, mais même un héros simplement

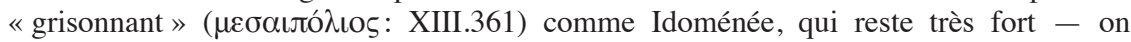

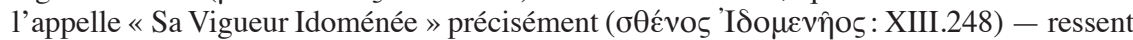
les effets de l'âge (cf. XIII.481-6).

25. Sur le contraste entre Nestor et Diomède dans cette scène, voir Richard MARTIN, The Language of Heroes: Speech and Performance in the Iliad, Ithaca, 1989, p. 24-6. Agamemnon décerne régulièrement à Nestor la palme à l'assemblée (cf. II.370). L'orateur de Pylos a toujours une idée, une $\mu \eta \hat{\imath \iota \varsigma, ~ e n ~ t e ̂ t e ~(c f . ~ 3.18) . ~}$ 


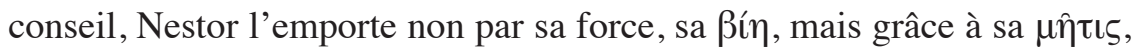
à ses «idées ». Or on ne peut trouver puissance plus contraire à la force brutale. La ßín et la $\mu$ îtı sont antinomiques. Leur affrontement est même « théogonique » si l'on en croit Hésiode ${ }^{26}$. Ici, elles sont simplement aussi opposées que la jeunesse et la vieillesse. Les jeunes guerriers se fient à

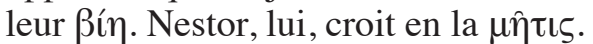

On voit bien dès lors l'étrangeté de la formule Пoıó $\mu$ oıo ßínv, « la force de Priam », appliquée par Ménélas au vieux souverain (III.105). Identifier un vieillard par ses avis, ses idées, sa $\mu \hat{\tau} \tau \iota \varsigma$, voilà la norme. Mais non par sa vigueur! L'Atride lui-même le désigne ensuite comme un vieil homme (III.109). Et nous apprenons un peu plus loin que l'âge précisément l'a écarté du combat ${ }^{27}$ et cantonné au « Conseil des Anciens », où seuls brillent les discours (III.149-51). Sa force ne réside plus dans ses membres, mais dans sa « voix au son doux comme le lys » (cf.ő $\pi \alpha \lambda \varepsilon \varepsilon \iota ı ́ \varepsilon \sigma \sigma \alpha v:$ III.152). Le parallèle avec Nestor est évident. Voici comment ce dernier nous est présenté pour la première fois dans l'Iliade :

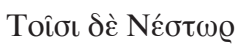

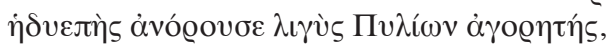

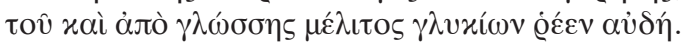

Devant eux Nestor

au suave langage se leva, l'orateur sonore de Pylos; depuis sa langue coulaient des accents plus doux que le miel. (I.247-9)

À eux deux, Nestor et Priam nous offrent dans l'Iliade le portrait vivant du bon roi idéal qui, dans la Théogonie, règle les conflits grâce aux dons oratoires qu'il a reçus des Muses. Les filles de $\mu \eta \tau i ́ \varepsilon \tau \alpha$ Zev́ et de Mémoire « ont versé sur sa langue une douce rosée » (Théogonie, 83). « Heureux celui que les Muses chérissent: de sa bouche coulent de doux accents!»

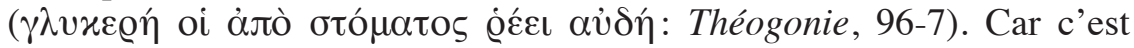
d'abord « de la bouche des Muses que coulent d'infatigables et suaves accents; et elle sourit, la demeure de leur père, Zeus aux éclats puissants, quand la voix douce comme le lys des déesses s'épand» (Théogonie,

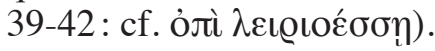

26. Sur la nature antinomique de la ßín et de la $\mu \hat{\tau} \tau \iota \varsigma$, cf. Marcel Detienne et JeanPierre Vernant, Les ruses de l'intelligence. La mètis des Grecs, 1974, en particulier la deuxième partie, p.61-125. Dans la Théogonie d'Hésiode, l'affrontement de ces deux puissances fonde le mythe de souveraineté qui finira par asseoir Zeus sur le trône (cf. Théogonie, 886-900).

27. Voir aussi au Chant XXIV la scène célèbre entre Priam et Achille où le vieux roi commence par évoquer le souvenir de Pélée et se compare à lui : tous deux ont même âge et sont « au seuil maudit de la vieillesse » (XXIV.486-7). 
La vieillesse confère au héros des talents « musicaux ». Lorsque Ménélas note qu'un vieillard sait rapprocher l'avenir du passé (III.109-10), il ne fait que prêter à Priam le talent naturel des Muses qui savent dire « ce qui est, ce qui sera, ce qui fut » (Théogonie, 38). En revanche, invoquer le vieux roi au nom de sa force, sa ßín, dont il est en réalité privé, et non pour son intelligence, sa $\mu \eta \hat{\imath \iota \varsigma, ~ q u i, ~ e l l e, ~ s ' a f f e r m i t ~ a v e c ~ l ' a ̂ g e ~}{ }^{28}$, reste un mystère.

\section{Le style formulaire}

Pour échapper à cette difficulté, la première explication qui vient à l'esprit tient évidemment à la nature formulaire de la langue homérique. Si l'on ne peut dire avec certitude dans quelles conditions l'Iliade et l'Odyssée, telles que nous les connaissons, ont été créées $^{29}$, on sait cependant, depuis les travaux de Milman Parry ${ }^{30}$, que ces épopées tirent leur origine d'une poésie orale, composée et recomposée, tant pour le fond que pour la forme, par des générations d'aèdes. Fondé sur un travail de mémoire, le chant procède suivant des thèmes connus de tous et répète des «formules » ciselées par la tradition ${ }^{31}$.

Dès lors, il devient aisé d'expliquer les quelques tours apparemment incongrus que l'on relève dans les poèmes homériques, comme « l'irréprochable Égisthe », selon la traduction consacrée ${ }^{32}$, au début de l'Odyssée (1.29). Mais aussi, pourquoi pas, « Sa Force Priam » qu'invoque Ménélas. En effet, si l'on suit ce raisonnement, le fils d'Atrée emploie ici une «formule » dans laquelle le mot «force »n'a pas son sens propre, mais intervient, associé au nom de Priam, en fonction des impératifs syntaxiques et métriques du moment, pour désigner, sur ce ton plein de majesté propre à l'épopée, la personne du vieux roi.

28. Cf. Detienne et Vernant, op. cit., p. 23-4.

29. Ce n'est pas ici le lieu d'un débat sur la question homérique. Pour un résumé récent, voir par exemple Robert Fowlen, « The Homeric Question », The Cambridge Companion to Homer, Cambridge, 2004, p. 220-32.

30. À commencer par sa thèse, L'épithète traditionnelle dans Homère: essai sur un problème de style homérique, Paris, 1928.

31. Cf. Milman PARry, The Making of Homeric Verse: the Collected Papers of Milman Parry, Oxford, 1971, en particulier p. 272.

32. Anne Amory PARRY a montré que cette traduction ne tient pas et que l'incongruité n'est qu'apparente: Blameless Aegisthus: A study of AMYMQN and other Homeric Epithets, Leiden, 1973. Sur ce point, voir aussi Steven LowENSTAM, «Irus'Queenly Mother and the Problem of the Irrational Use of Homeric Epithets », Pacific Coast Philology 16, $\mathrm{n}^{\circ} 1,1981$, p. 39-47. 
Pourtant, cette conclusion n'est pas entièrement satisfaisante. Comme l'a noté le fils de Milman Parry, Adam Parry ${ }^{33}$, expliquer uniquement de la sorte les formules qui sont la matière même de la poésie homérique nous fait courir le risque d'ôter à cette dernière une large part de sa qualité littéraire en supprimant toute originalité ainsi que toute pertinence dans le choix des sujets, des thèmes et des termes, qui ne sont plus conçus que comme le produit des contraintes formelles et culturelles auxquelles l'aède est soumis ${ }^{34}$. Les mots mêmes qui forment le texte sont menacés de perdre leur sens précis, tout particulièrement quand il s'agit des plus traditionnels d'entre eux, les épithètes formulaires ${ }^{35}$. De sorte qu'il devient vite impossible de tirer un sens des jeux d'échos qui abondent, évidemment, dans une telle œuvre.

Mais en réalité, il est permis d'accorder une signification aux liens qui unissent les formules entre elles. Comme le note Pietro Pucci, « la répétition de vers et d'expressions peut faire sens par les effets intertextuels qu'elle produit $\aleph^{36}$. Les formules ne sont pas des expressions génériques répétées selon la seule nécessité du mètre. Elles sont pertinentes au contraire. La notion de «thème » est ici essentielle ${ }^{37}$. Loin d'être un obstacle à la richesse et à la qualité du chant, le jeu formulaire est leur instrument. Norman Austin retient la métaphore musicale pour décrire ces « éléments fondamentaux vers lesquels Homère revient encore et encore, comme

33. Adam PARry, The Language of Achilles, Oxford, 1989.

34. La manière dont Adam Parry a résumé et introduit l'œuvre de son père fait ellemême l'objet de critiques: cf. Gregory NAGY, Homeric Responses, Austin, 2003, p. 60 sq. Reste que les jeux d'écho tissés par le jeu formulaire sont significatifs et méritent d'être relevés et étudiés. Pour d'utiles clarifications sur la perspective « oraliste », voir Albert Lord, The Singer Resumes the Tale, Ithaca, 1995, p. 1-95; voir aussi Richard JANKO, « The Homeric Poems as Oral Dictated Texts », The Classical Quarterly N.S.48, n 1, 1998, p. 1-13; Gregory NAGY, Greek Mythology and Poetics, 1990, Ithaca-Londres, p. 18-35 et Homeric Questions, Austin, 1996, chapitre 1.

35. PARry, The Language of Achilles cité, p. 254, note 99. Voir aussi p. 256-7, en particulier la note 105, sur l'idée qu'un épisode chasse l'autre.

36. Pietro Pucci, Ulysse polutropos, Lille, 1995, p. 342; voir aussi p. 51-2. Sur « intertextualité » et «oralité », cf. NAGY, The Best of the Acheans cité, préface à l'édition de 1999, p. XIv, § 22 sq.; Homeric Responses cité, p. 8-19; Christos Tsagalis, The Oral Palimpsest, Harvard, 2008.

37. Aux yeux de Nagy (Homeric Questions cité), cette conception ne contredit pas les conclusions qu'il juge les plus importantes des travaux de Parry et Lord. Pour Milman Parry, en effet, l'association de mots produisant une formule se fait en accord avec « un schéma de pensée donné » (The Making of Homeric Verse cité, p. 270), schéma que Nagy assimile à un thème précisément, contenu traditionnel à partir duquel s'élabore le chant nouveau. Quant à Albert Lord, la notion de «thème » est au centre de ses réflexions. 
un compositeur de musique peut revenir encore et encore à sa gamme fondamentale et à ses thèmes $»^{38}$.

Si l'on adopte cette perspective, qui permet de tenir compte du rôle joué par la tradition dans la composition des poèmes homériques, sans pour autant priver ces œuvres de leur originalité ${ }^{39}$ et de leurs qualités proprement littéraires, on ne peut plus aussi aisément écarter les apparentes impropriétés que présente parfois telle ou telle formule. Il est donc temps désormais de revenir à « la force de Priam », pour essayer de comprendre comment cette formule s'inscrit dans l'économie générale de l'œuvre. Son impertinence est-elle réelle ? Est-ce un simple titre de noblesse accordé à Priam par Ménélas, en accord avec le ton héroïque du poème, mais sans lien avec le contexte narratif? Ou bien cette formule est-elle significative?

\section{Une formule archaïque}

Notons d'abord que notre formule est, d'un point de vue formel, assez remarquable. Désigner un héros par sa vigueur est une pratique très ancienne. Elle remonte à l'indo-européen et l'on trouve des équivalents du tour associant un mot qui signifie la force à une épithète formée sur le nom du héros en sanskrit par exemple ${ }^{40}$. Le caractère archaïque de cette formule ${ }^{41}$ explique donc qu'elle se présente, d'un point de vue linguistique, sous un jour particulier.

Milman Parry s'est d'abord intéressé aux noms propres, dont l'emploi fréquent ainsi que la fixité relative - un nom commun, lui, peut présenter de nombreux synonymes - rendait l'étude particulièrement pertinente, les variations constatées portant essentiellement sur les adjectifs qui les

38. Norman Austin, Archery at the Dark of the Moon: Poetic Problems in Homer's Odyssey, University of California, Berkeley, 1975, p. 6.

39. Sur l'originalité de l'Iliade et de l'Odyssée par rapport aux autres poèmes du Cycle en particulier, voir par exemple Jasper GRIFFIN, «The Epic Cycle and the Uniqueness of Homer », Journal of Hellenic Studies 97, 1977, p. 39-53; et plus récemment Jonathan S. Burgess, The Tradition of the Trojan War in Homer \& the Epic Cycle, Baltimore-Londres, 2001.

40. Le linguiste José Luis GARCIA RAMÓN revient sur cette formule dans un article récent: «Homme comme force, force d'homme: un motif onomastique et l'étymologie du vieil irlandais gus », Actes du Colloque de travail de la Société des Études Indo-Européennes, Paris, 22-24 octobre 2004, Georges-Jean Pinault et Daniel Petit (éd.), Paris, 2006, p. 79-93. L'ouvrage de référence reste celui de Rüdiger Sснміт, Dichtung und Dichtersprache in indogermanischer Zeit, Wiesbaden, 1967, p. 109-11. La variante consistant à employer le génitif du nom du héros lui-même est moins archaïque: ibid., p. 110, note 670.

41. G. S. KIRK parle d'un «idiome ancien »: The Iliad: A Commentary, Vol. I, Books 1-4, Cambridge, 1985, p. 226, à propos de II.658. 
accompagnent. Syntaxiquement, ces formules se présentent toujours de même: le nom du héros est qualifié par une épithète et forme avec elle une dénomination plus ou moins caractéristique. Parry distingue ce qu'il appelle les « épithètes génériques », qui s'appliquent à divers personnages, et les « épithètes spéciales », qui ne vont qu'à un seul héros.

Dans le cas de « la force de Priam» toutefois, le tour est différent. Le terme qui note la qualité attribuée au héros et qui sert habituellement d'épithète dans la formule est ici le substantif: ßín; et c'est le nom du

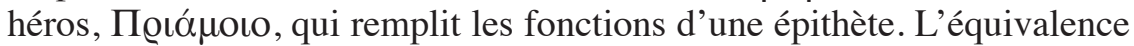
syntaxique entre le génitif déterminatif et l'épithète correspondante apparaît clairement dans la formule originale appliquée à Héraklès où les

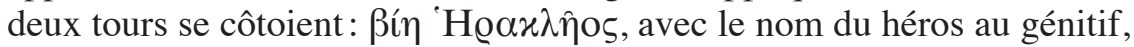

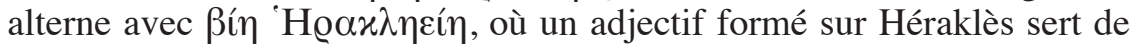
qualificatif. Gregory Nagy a travaillé sur cette formule ${ }^{42}$. Il note comment, à travers cette périphrase, c'est la force elle-même, la ßín, qui apparaît comme l'un des éléments essentiels de l'épopée d'Héraklès ${ }^{43}$ et, plus généralement, comme un «thème épique traditionnel » à partir duquel

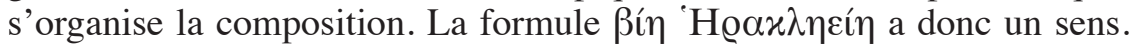
Est-ce dû seulement au fait qu'appliquée à Héraklès nous l'avons sous sa forme originale? Pour l'avoir déjà croisée à propos de Diomède et de Priam, nous savons qu'elle n'est pas « spéciale » selon les critères de Milman Parry. Pourtant, elle n'a rien non plus de « générique ». Elle est au contraire tout à fait significative.

Comme le remarque Gregory Nagy, ce n'est pas un hasard si cette formule se retrouve d'abord appliquée à des héros dont le nom même rappelle celui d'Héraklès et, à travers lui, le $x \lambda \hat{\varepsilon}$ os épique. En effet, dans l'Iliade,

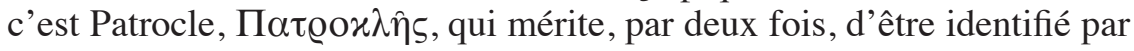
sa force, alors que son meurtrier et les armes d'Achille occupent le devant de la scène. Au Chant XVII d'abord, Hector s'apprête à revêtir l'armure dont il a dépouillé le compagnon d'Achille, « Sa Force Patrocle », après

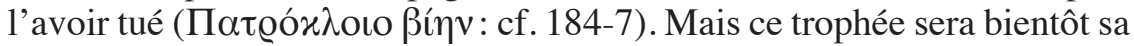
propre ruine (cf. 188-211) et, au Chant XXII, Achille, tout à sa vengeance, n'a d'yeux précisément que pour ces armes que le Priamide a arrachées à son ami (XXII.323 = XVII.187). Que l'Iliade insiste ainsi sur le fait qu'Hector a tué «Sa Force Patrocle » n'est pas fortuit. Non seulement cette mention, pour la forme et les sonorités, correspond de très près à

42. Cf. NAGY, The Best of the Acheans cité, p. 318-9.

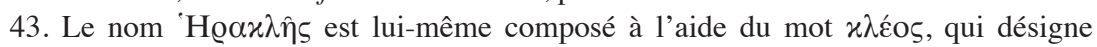
traditionnellement chez Homère à la fois la gloire du héros, mais aussi, au-delà, le chant

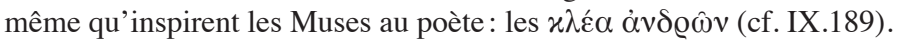


la formule originale appliquée à Héraklès, mais elle fait aussi sens dans l'économie générale du poème. En effet, si elle grandit l'exploit du fils de Priam, elle l'inscrit surtout dans l'affrontement attendu entre le « meilleur des Achéens », Achille, et le meilleur des Troyens, Hector, affrontement qui, dans l'épopée, doit justement être décidé par la force (XV.613-4:

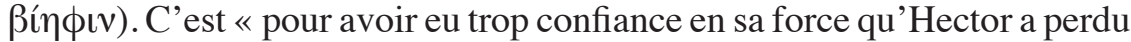

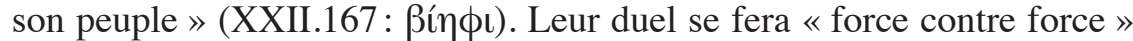

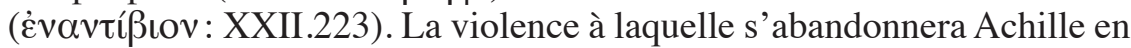
outrageant la dépouille de son adversaire donnera raison à Polydamas, qui craignait que la mort de Patrocle aux mains d'Hector ne réveille « le cœur

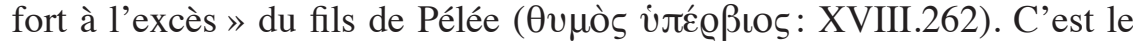
visage blâmable de la $\beta$ í $\eta$, comme en témoignent les critiques que formule Apollon contre l'attitude d'Achille à la fin de l'épopée ${ }^{44}$.

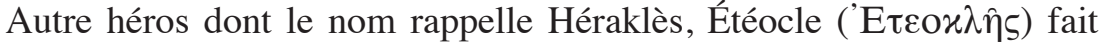
une brève apparition au Chant IV. Agamemnon, pour exciter Diomède au combat, lui oppose le souvenir de Tydée, son père, si brave et si fort. Le compagnon de Polynice, porteur d'un message de son camp, s'est rendu, seul, dans la demeure où régnait « la force d'Étéocle » (IV.396). La conclusion de l'Atride est éclairante et donne tout son sens à l'emploi de cette formule: Diomède vaut moins que Tydée au combat, s'il est meilleur à l'assemblée (IV.399-400). Nous retrouvons bien ici les termes de l'opposition traditionnelle entre la force et l'idée.

Enfin, dans l'Odyssée, c'est Iphiklos que désigne ce tour (XI.290; XI.296), avec ici deux jeux de mots supplémentaires. D'abord, s'il s'agit ici du fils de Phylakos ${ }^{45}$ et non d'Iphiklès, fils d'Amphitryon et frère d'Héraklès, l'homonymie présente dans l'épithète, elle, renforce la pertinence de la formule. Mais surtout, ce personnage est doublement fort, son nom même étant formé à partir de î $\phi$, instrumental de ǐs, doublon de ßí est comparable à celui que l'on relève au vers 332 de la Théogonie où Héraklès est appelé «la vigueur de la force d'Héraklès » (ís [...] ßín

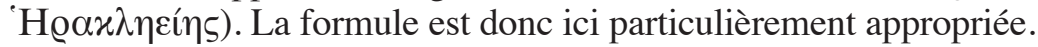

Dans tous ces exemples, il est aisé de voir comment le jeu formulaire permet d'expliquer l'usage de cette périphrase à la fois expressive et significative. Mais qu'en est-il des personnages dont le nom ne rime pas avec celui du fils de Zeus? Si l'on peut encore faire jouer les impératifs métriques pour expliquer le choix de cette formule particulière, on ne sera

44. Cf. XXIV.32-54; en particulier la comparaison des vers 39-44.

45. Il apparaît brièvement dans l'Iliade à l'occasion du catalogue des vaisseaux (II.705). Pour plus de détails sur ce personnage, cf. Timothy GANTZ, Early Greek Myth, BaltimoreLondres, 1993, vol. I, p. 185-7 (trad. Mythes de la Grèce archaïque, Paris, 2004). 
plus en mesure de faire entrer la composition de leur nom dans le sens qu'on lui attribue. Et, dès lors, le risque d'impropriété grandit considérablement. Mais en réalité, il n'en est rien.

Mise à part «la force de Priam » que convoque Ménélas au Chant III et qui pose problème, les autres emplois de cette formule dans l'Iliade viennent à propos. Ainsi celui qui concerne Diomède au Chant $\mathrm{V}$, en pleine

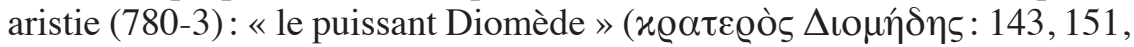

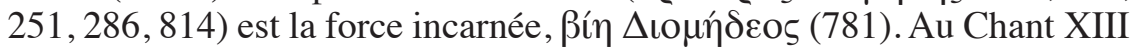
$(758,770,781)$, cette formule concerne un fils de Priam, Hélénos, connu pour être « de beaucoup le meilleur des devins » (VI.76), un homme qui comprend le vouloir des dieux (VII.44-5). Mais ici, il est d'abord l'un des champions troyens partis à l'assaut du rempart qui protège le camp achéen (XII.88-95), le meurtrier de Déipyre (XIII.576-80), l'adversaire malheureux, mais courageux, de Ménélas, qui le blesse à la main à l'issue de leur duel (581-601). Citons encore «la force d'Énée», appelée à remplacer à la tête de Troie la race de Priam (XX.306-8). Ou « la force de sire Teucros $»^{46}$ (XXIII.859), qui se lève afin de prendre part au concours de tir à l'arc organisé par Achille en l'honneur des funérailles de Patrocle et décoche le premier trait «puissamment ( $\dot{\varepsilon} \pi \iota x \varrho \alpha \tau \dot{\varepsilon} \omega \varsigma: 863)$. Tous ces usages sont manifestement pertinents.

Enfin - c'est pour nous l'emploi le plus intéressant, car placé dans la bouche du même Ménélas qui convoquait tantôt « la force de Priam»-, au Chant XVII, l'Atride affronte Euphorbe autour du cadavre de Patrocle. Ce jeune Troyen fut le premier à lancer sa pique dans le dos du fils de Ménœetios, désarmé par Apollon, avant de prendre la fuite son forfait accompli (XVI.806-15). Il se vante de cet «exploit» devant Ménélas (XVII.14-5). Or Euphorbe rappelle la figure de Pâris ${ }^{47}$, autre éphèbe meilleur aux jeux qu'à la guerre, autre combattant de loin plutôt que de près, autre ennemi juré de Ménélas, instrument surtout de la mort à venir d'Achille quand Apollon dirigera son trait fatal sur le talon du Péléide. De même, son frère, Hypérénor, que Ménélas a tué à la fin du Chant XIV (516-9), était lui aussi un jouvenceau prompt à outrager le fils d'Atrée. Il est donc tout naturel que la réponse de l'Atride à Euphorbe (XVII.1932) fasse écho à sa tirade du Chant III (les vers 106-8 en particulier),

46. Teucros, s'il est expert à l'arc, est aussi un homme fort et vaillant: cf. XIII.314.

47. Sur ce parallèle, cf. Richard JANKo, The Iliad: A Commentary, Vol. IV, Books 13-16, Cambridge, 1992, p. 414. Les arguments en faveur du rapprochement entre Euphorbe et Pâris me paraissent bien plus forts que ceux qui les séparent; pour le sentiment contraire, cf. William Allan, «Arms and the Man : Euphorbus, Hector, and the Death of Patroclus », Classical Quarterly N.S.55.1, 2005, p. 1-16 (avec une bonne bibliographie). 
notamment dans la critique qu'il livre de la jeunesse troyenne, vantarde et livrée aux excès (XVII.29; voir aussi 23) ${ }^{48}$, puérile et sotte également (cf. vท́лıо : 32). Pourtant, cette fois, force et jeunesse sont bien associées dans la bouche de Ménélas (24-5). La fougue accompagne Euphorbe et Hypérénor (cf. 20-3 et 29), deux jeunes guerriers, comme il se doit, et non un vieillard. Les formes sont respectées; la formule aussi.

Nous ne pouvons toutefois pas encore conclure que le cas de Priam est bien à part. Pour être complets, il nous faut d'abord procéder au relevé des différentes formules qui, reposant sur d'autres substantifs que ßí $\eta$, mais synonymes de ce dernier, se substituent, en fonction des impératifs métriques, à l'objet de notre étude. C'est là la règle du jeu formulaire.

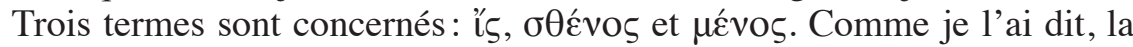
synonymie n'est pas parfaite. Ainsi, outre que seul le mot ßín comporte régulièrement une idée de violence et de contrainte et présente la force sous ses deux visages, l'un louable, l'autre blâmable, il n'est jamais employé pour décrire la force d'un élément naturel, mais toujours d'un homme ou d'un animal. Au rebours, ce sont là des emplois courants pour ces autres termes, qu'il s'agisse de la force du vent (XV.383; XVII.739; etc.), d'un fleuve (XXI.356 et 383), de l'océan (XVIII.607; XXI.195), du soleil (XXIII.190; 10.160; 12.175-6), d'un astre (XVIII.486). En outre, plutôt que d'y lire une figure de style, on peut souvent comprendre ces expressions littéralement. Autrement dit, ces tours n'ont pas tout à fait la même solennité que la formule d'origine.

Ils trouvent toutefois des usages comparables, appliqués à divers héros effectivement forts et généralement dans des contextes où cette qualité est mise à l'épreuve. Un seul, pour les sonorités, rappelle le nom d'Héraklès: au Chant XVI de l'Iliade, nous découvrons l'histoire de « Sa Puissante Fougue Échéklès » (189). Mais nous croisons aussi « Sa Vigueur Idoménée » (XIII.248) dans ce qui forme la longue aristie du roi crétois. Au Chant XIV, quand Ajax l'a atteint d'un jet de pierre, « bien vite Sa Fougue Hector est tombé par terre, dans la poussière » (418). Lors des jeux en l'honneur des funérailles de Patrocle, Ulysse et Ajax s'affrontent à la lutte. Aucun des deux n'a l'avantage. Si Ulysse n'est pas capable d'amener Ajax à terre, le fils de Télamon ne l'est pas davantage: " Sa Puissante Force Ulysse tenait bon » $(\text { XXIII.720 })^{49}$. Enfin, toujours à l'occasion de ces jeux, on nous parle de «Sa Grande Vigueur Éétion » (XXIII.827), tué au combat

48. Sur les reproches qu'adresse Ménélas aux « Troyens insolents », voir aussi XIII.620 sq.

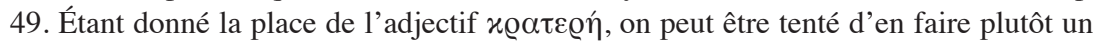
attribut, ce qui pourrait donner en français: « Sa Force Ulysse résistait puissamment ». 
par le divin Achille aux pieds infatigables qui lui a ravi l'énorme bloc de fer que doit désormais lancer « Sa Puissante Fougue Léontès » (837) s’il veut remporter le concours ${ }^{50}$. Rien d'impertinent dans ces désignations: tous ces héros sont vigoureux et s'illustrent dans des contextes où la force prime.

Nous trouvons en outre une mention notable de « la vigueur d'Hector» dans la bouche d'Achille. L'Atride est incapable de contenir « la vigueur d'Hector meurtrier » (IX.351). Ici, l'expression est plus aisément rendue par un tour littéral que par la formule consacrée («Sa Force Hector»). Comme quand au Chant VII Apollon propose d'exciter «la puissante fougue d'Hector dompteur de cavales » (38) pour qu'il lance un défi aux preux du camp grec, on peut entendre cet exemple plus proprement. Hector est bien désigné par sa force, mais le fait que le nom du héros soit lui-même accompagné d'une épithète traditionnelle déplace l'accent du substantif vers son complément, de la vigueur vers son détenteur ${ }^{51}$. Ce glissement devient complet quand la force se trouve associée dans un hendiadys avec les bras du héros et ne rentre donc plus vraiment dans la dénomination qui nous intéresse (XVIII.638) $)^{52}$.

Pour finir, si nous relevons les exemples présents dans l'Odyssée, il apparaît que, par rapport à l'Iliade, l'usage de cette formule est un peu galvaudé. Ainsi, on trouve pas moins de sept occurrences de «Sa Sainte Force Télémaque » $(2.409 ; 16.476$; etc. $)$ et onze de « Sa Fougue Alkinoos » (parfois «sainte» elle aussi, parfois non: $7.167 ; 8.2$; etc.). À deux lettres près, celle-ci revient même une douzième fois pour désigner Antinoos

50. Juste après a lieu le concours de tir à l'arc auquel participe «la force de sire Teucros » (XXIII.859).

51. De la même manière, au Chant XI, les deux mentions de «la fougue de l'Atride »

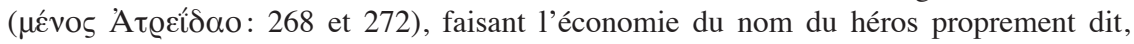
Agamemnon, ne me semble pas relever de notre formule. Au Chant XXI, le fleuve Scamandre propose à son frère le Simoïs de l'aider à contenir « la vigueur du héros » (3089), mais ne mentionne pas nommément Achille. Citons encore, parmi ces exemples un peu à part, les deux emplois de «la fougue d'Arès » (XVIII.264 et 16.269) où l'expression est mise pour «la guerre, le combat». Le contraste est net avec cet autre exemple divin mettant en scène «Sa Force Héphaïstos » au moment où il délivre des rets infrangibles qu'il a forgés son épouse infidèle, Aphrodite, et son amant, Arès (13.359). Ici c'est bien la vigueur du dieu (celle de ses bras comme de ses idées) qui est mise en exergue face à la déloyauté de sa femme.

52. Voir aussi VII.309: Hector, à l'issue de son duel avec le fils de Télamon, a échappé à « la fougue et aux bras intouchables d'Ajax ». On voit bien ici les jeux d'échos que produit le style formulaire: le duel s'ouvrait sur Apollon excitant la fougue d'Hector (VII.38) et se clôt sur Hector échappant à la fougue d'Ajax. La boucle est bouclée. 
(18.34). Reste que Télémaque et Antinoos sont jeunes et vigoureux. La brutalité et la violence des prétendants sont bien connues (cf. vं $\tilde{\varepsilon} Q \beta$ ¿ov: $14.92 ; 14.95 ; 16.315 ; 16.410)$. Télémaque, lui, a hérité la vigueur de son père, comme le prouve le fameux concours de tir à l'arc (cf. 21.126-7) ${ }^{53}$. Alkinoos enfin, s'il est plus âgé, n'est nulle part décrit comme un vieillard.

Une fois tous les exemples disséqués, notre étrange formule, « la force de Priam », reste donc seule inexpliquée, voire, en apparence, inappropriée. On peut bien avancer qu'elle seule repose uniquement sur les contraintes formulaires, qu'elle seule n'a pas de « sens ». Mais ce n'est guère convaincant. Mieux vaut donc considérer que Ménélas choisit bien ses mots.

\section{Le chasseur noir}

Au Chant III, le discours de l'Atride est bâti autour de l'opposition entre Priam et ses fils. Sans doute le moment est-il venu de nous intéresser à eux. En effet, si la formule «Sa Force Priam » est inattendue, le paradoxe ne fait que croître quand le fils d'Atrée décrit les fils du souverain troyen.

De la force, de la vaillance des Priamides, apanage traditionnel de la jeunesse, Ménélas ne fait nulle mention. Ils sont jeunes, assurément, mais leur âge n'est pas le gage ici d'une fougue guerrière, d'un bras puissant: il n'est question que de leur esprit, bien léger (III.108). On ne peut se fier à eux : ils sont «arrogants et déloyaux »(III.106). Ce sont là des traits qui ne se rapportent pas à la force, mais à la ruse au contraire. La ßín est régulièrement condamnée dans l'Iliade lorsqu'elle se fait violence. Mais

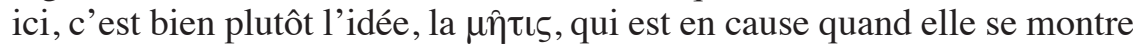
infidèle et trop sûre d'elle ${ }^{54}$. Priam est convoqué au nom de sa force, ses fils dénoncés pour leurs dangereuses idées : c'est le monde à l'envers !

Le premier visé par Ménélas est évidemment Pâris. Nous pourrions donc nous dire que nous avons affaire aux railleries d'un mari en colère. Mais le ton paraît bien solennel. Ménélas est un personnage grave. Il tient régulièrement pour l'honneur, la vertu, le droit même, la $\theta \dot{\varepsilon} \mu \varsigma^{55}$. Ramener

53. Les prétendants sont les premiers à reconnaître qu'il s'agit là d'une épreuve de force dans laquelle le souvenir des exploits d'Ulysse sert de référence (21.91-4). Eurymaque, dépité, le confirme un peu plus loin: « Je suis confus que pour la force (ßínऽ) nous soyons si inférieurs | au divin Ulysse, puisque nous sommes incapables de tendre | 1'arc!» (21.253-5).

54. Cf. Detienne et Vernant, op. cit., p. 23-4.

55. Cf. XIII.620-39; XXIII.570-85. C'est en particulier l'interprétation que donne de ce personnage Philippe Rousseau, pour qui il incarne la légitimité et le souci de cohésion de la communauté: "Le deuxième Atride: le type épique de Ménélas dans l'Iliade », dans Évelyne Geny et Marie-Madeleine Mactoux (éd.), Mélanges Pierre Lévêque vol.5, 
la pertinence de ses propos à la simple jalousie n'est pas suffisant. En outre, dans l'épopée, les discours des héros doivent être ratifiés par les événements - et donc, en dernier ressort, par le Ciel qui décide de leur issue - pour avoir du poids; sinon, ils restent un vain mot. Or, loin de ne trouver confirmation que dans le ressentiment de l'Atride envers Alexandre, les attaques de Ménélas s'appuient sur de nombreux jeux d'écho dans le reste de l'Iliade.

Car Pâris est effectivement dénué de force et de vaillance. Ce n'est pas là seulement une injure de son rival. Hector même le lui reprochait tantôt: à le voir si beau, on se figure un preux, «alors qu'au fond de lui, il n'est

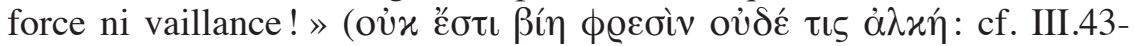
5; voir aussi VI.521-3). L'apparence est trompeuse. Sous son éclatante beauté, c'est un fanfaron sans vigueur ni courage. Il n'aime pas l'épreuve de force. Pire, quand il participe à la bataille, il préfère agir en traître. Ainsi, au Chant XI, « embusqué », il ne «bondit hors de sa cachette » (ं் $x$

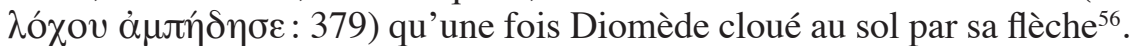
C'est pourquoi, aux yeux du fils de Tydée, ses « vantardises » $(379,388)$ sont autant d'enfantillages et méritent le mépris :

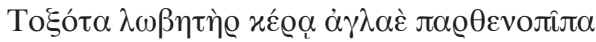

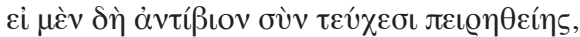

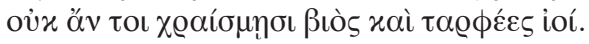

Ah! l'archer! l'insulteur! l'homme fier de son arc! le lorgneur de fille!

si tu me venais tâter face à face, en armes,

ce n'est plus ton arc, ta provision de flèches qui te serviraient de rien. (XI.385-7)

Ici s'affrontent le héros hors des lignes, ancêtre de l'hoplite, et l'archer. Cette opposition est classique ${ }^{57}$. Pâris est le prototype du « chasseur noir » décrit par Pierre Vidal-Naquet dans son célèbre article: c'est un « éphèbe inachevé $\gg^{58}$. Aux yeux de Diomède, il agit en gamin insensé (388-90). Il

Besançon, 1990, p. 325-54; «Remarques complémentaires sur la royauté de Ménélas », dans Michel Woronoff (éd.), L'univers épique, Paris, 1992, p. 57-79; voir aussi sa thèse

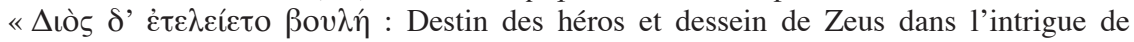
l'Iliade », Lille, 1996.

56. Cette scène évoque évidemment la manière dont Pâris, aidé d'Apollon, tuera plus tard Achille, même si l'Iliade ne raconte pas directement la mort du Péléide.

57. Cf. Sophocle, Ajax, 1120-3; Euripide, Héraklès, 151-64: même la valeur d'un héros aussi fort qu'Héraklès devient suspecte, dès lors qu'il se bat avec un arc. Voir Pierre VIDALNAQUET, Le chasseur noir, Paris, 1981, p. 169, note 76.

58. Pierre Vidal-Naquet ne prend pas Pâris pour exemple dans l'article original (Le chasseur noir cité, p. 151-76), mais il le fait dans Le monde d'Homère, Paris, 2000, p. 107-8. 
ne se bat pas en héros : il épie, il chasse son adversaire. Et non comme un chasseur valeureux qui agit en plein jour, muni d'une pique, et affronte sa proie de face ${ }^{59}$ : Alexandre est un chasseur à $l^{\prime}$ 'affût ${ }^{60}$, qui piège ses ennemis .

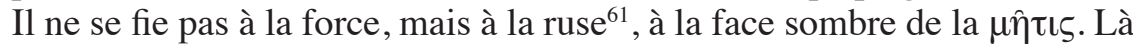
où un brave rencontre l'ennemi au « corps à corps », « force contre force »

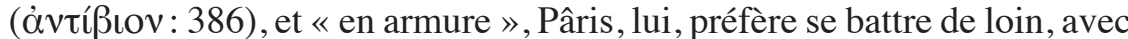
« un arc et des flèches » (387). Dans l'Iliade, c'est le signe certain qu'il ne compte pas parmi les preux.

Car les archers n'ont pas bonne réputation dans l'épopée d'Achille, héros fort entre tous, ennemi de la fourbe et du mensonge (cf. IX.308 sq.). À l'issue du duel entre Ménélas et Pâris, c'est Pandare, l'archer expert (V.245), qui vient confirmer toutes les préventions de l'Atride à l'égard de ses adversaires. En rompant la trêve et en tentant de tuer Ménélas d'une flèche, le fils de Lycaon ratifie le discours du fils d'Atrée: ce sont bien les Troyens qui « les premiers, en passant outre, ont ruiné le pacte loyal » (IV.236; cf. IV.67, 72 et 155), ainsi que Ménélas le craignait dans notre extrait (III.107). De plus, non contents de frapper en traître, tels des chasseurs en embuscade - l'arc même de Pandare est le produit d'une telle chasse (IV.105-11) —, les archers manquent de courage (cf. XIII.713 sq.). Confiants non dans leurs propres forces, mais dans leurs arcs, ils tirent « depuis l'arrière », " sans être vus ». L'oubli est leur arme. Ils font perdre à l'adversaire la mémoire de son ardeur au combat. Bref, ils sont une menace pour l'héroïsme même. L'arc est une arme maudite. Au Chant V, Pandare se fie au sien (205). Pourtant, une fois le fils de Lycaon isolé sur le champ de bataille face à Diomède furieux, son arc ne lui sert plus à rien $(205,214-6)$. Énée le lui dit bien : pour affronter le fils

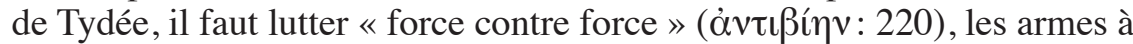

59. Pour un exemple classique dans l'Iliade même, on relira la légende de Méléagre que raconte le vieux Phénix lors de l'ambassade (IX.524 sq.). Cf. VIDAL-NAQUET, Le chasseur noir cité, p. 170.

60. Sur ce point, voir VIDAL-NAQUET, Le chasseur noir cité, p. 169 sq.

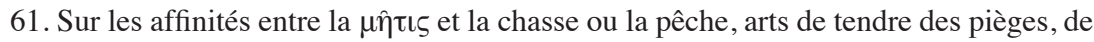
prendre dans des rets, cf. Detienne et Vernant, op. cit., p. 35-40, 52-4, 230-1. Diomède participe lui aussi à un épisode que l'on a comparé à une chasse nocturne: la Dolonie (cf. Vidal-NAquet, Le chasseur noir cité, p. 159-60). Dans la pensée archaïque, chasser en usant de $\mu \hat{\eta} \tau ı \varsigma$ n'est pas un crime en soi. Ce n'est que lorsque la ruse agit sans vigueur et fait ombrage à la valeur qu'elle mérite le blâme, et non systématiquement. Idoménée,

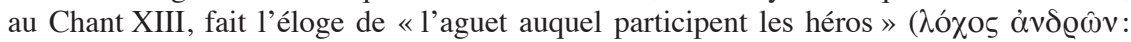
285; voir aussi 277) et qui regroupe tous les meilleurs soldats ( «l'aguet » où brille la valeur et où se distinguent le lâche comme le brave (277-8). Seule

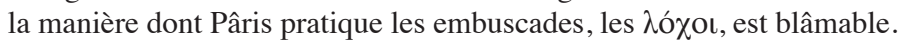


la main. Pandare renonce donc à son arc et adopte « la lance aiguë » (238, 279) d'un véritable guerrier. Mais c'est évidemment Diomède le plus fort à ce jeu-là. Pandare, lui, y laisse la vie (275-96): on ne s'improvise pas héros hors des lignes.

Certes, tous les archers ne sont pas sans valeur. Dans l'Iliade, il en est un qui se distingue particulièrement, Teucros, qui a reçu son arc d'Apollon en personne ${ }^{62}$. Au Chant XV, il forme avec son frère Ajax la dernière ligne de défense face aux Troyens lancés à l'attaque des nefs. Mais, si ses traits déciment l'ennemi (442-53), ils ne peuvent rien contre Hector lui-même: Zeus intervient et brise la corde de son arc plutôt que de le laisser frapper le fils de Priam (458-70). Ajax alors recommande à son frère de quitter son rôle d'archer et d'endosser les armes d'un preux (472-83) ${ }^{63}$. Cette transformation de l'archer, par ailleurs excellent, en valeureux guerrier est remarquable. Le fait de s'armer est opposé à l'usage de l'arc. Teucros doit quitter ses flèches pour pouvoir devenir un champion en armure, muni d'une « vaillante pique » (482) dont le maniement repose sur la vigueur des bras (474). Dans sa forme, cette scène d'armement est typique, mais le contexte en est remarquable. En particulier si on la rapproche de celle qui voit Pâris se harnacher pour son duel avec Ménélas.

Au début du Chant III, le Priamide n'est pas armé en guerrier, quand bien même il apparaît en première ligne (16-20). Son équipement est disparate: il porte à la fois les armes d'un preux (épée, piques ${ }^{64}$ ) et celles d'un archer. Il n'a pas de jambières, ni de bouclier. Nulle mention d'un casque, ni d'une cuirasse. Il est armé à la légère et couvert d'une peau de panthère qui nous renvoie clairement à l'univers de la chasse. En outre, son attitude est comparable à celle d'un archer et non d'un véritable guerrier. Car son cœur ne tient pas quand il faut lutter de pied ferme ${ }^{65}$. Dès que Pâris voit Ménélas bondir à terre pour l'affronter (29), son cœur est frappé de crainte

62. Cf. XV.440-1. L'Iliade fait, en passant, une autre exception pour « Philoctète expert à l'arc » (II.718), qui tient son arme d'Héraklès, et ses hommes « experts à l'arc pour com-

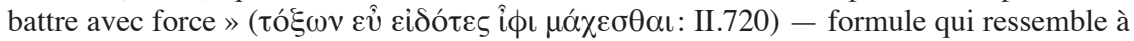
un oxymore. C'est que, traditionnellement, Philoctète est un archer valeureux qui déteste la ruse et le mensonge: cf. Sophocle, Philoctète, 254-458.

63. En temps normal, Teucros profite de la protection que lui offre le fameux bouclier de son frère pour se battre avec son arc en première ligne: c'est l'équivalent d'une chasse vaillante, cf. VIII.266 sq.

64. Kirk note que la mention de deux piques est curieuse et qu'il ne s'agit peut-être pas là de la lourde pique du guerrier qui ne quitte pas sa main, mais de javelines destinées à être lancées: op. cit., (supra, n. 41), p. 268. Il revient aussi sur la surprise que produit l'accoutrement de Pâris (p. 267-8).

65. Cf. XIII.713. 
(31). Terrifié par l'Atride, Alexandre va se réfugier dans les rangs troyens (36-7). Il faut les railleries d'Hector pour le convaincre d'affronter Ménélas en duel (38-57). Quant à la scène d'armement qui précède le combat, elle vient confirmer l'impression de départ. Alors que Ménélas, depuis le début du Chant, est « en armes » (29), comme tout héros qui se respecte, même s'il fait alors des préparatifs supplémentaires (339), Pâris, lui, jusque-là, ne se présentait pas en preux. Teucros tantôt n'avait besoin que d'un bouclier, d'un casque et de sa pique. Alexandre, lui, doit tout revêtir (328-38). Pire, il lui faut même emprunter la cuirasse de son frère Lycaon (333), car il est venu se battre sans! Ce n'est qu'après que le Priamide fait vraiment figure de guerrier. Lui et Ménélas offrent un spectacle terrifiant (340-3). Néanmoins, même alors, dans le cas d'Alexandre, il s'agit moins d'une réalité que d'une apparence. Le diagnostic d'Hector était juste. À le voir ainsi armé, on se figure un champion. Mais au fond, il n'est pas de force. Le Priamide s'est déguisé en valeureux héros le temps du duel, mais ce n'est pas là sa véritable nature. Sans surprise, le plus puissant est sans conteste Ménélas (92). Zeus même ratifie sa victoire (IV.13). Pâris, lui, ne doit la vie qu'à l'intervention d'Aphrodite, sa patronne (III.369-82).

Ce n'est pas un hasard si la seule qualité que Pâris semble tirer de sa jeunesse est sa beauté, ses talents de séducteur. Il ne se fie ni à ses forces, comme les jeunes guerriers qu'envie Nestor, ni aux bonnes idées, celles qui sont fermes, fidèles, salutaires : lui s'en remet à son éclatante beauté (cf. VI.510), aux «dons charmants d'Aphrodite d'or» (III.64). Qui se ressemble s'assemble: Pâris est «sans force ni vaillance » (III.45); Aphrodite également (cf. óv $\alpha \lambda x \iota \varsigma$ $\theta \varepsilon$ ós: V.331). En revanche, tous deux brillent par la ruse, la tromperie, la séduction. Privés de vigueur, sans ßín, ils se fient à la $\mu \hat{\imath} \tau ı \varsigma$. Mais ce n'est pas celle de Nestor, ni celle de Priam, sage, pondérée, dense. La leur est légère, instable, infidèle.

\section{Les dons charmants d'Aphrodite}

Quand Hector reprend son frère au début du Chant III (39-57), toute son ire est dirigée contre la beauté trompeuse et suborneuse de Pâris : elle fait croire à sa valeur quand Alexandre est «sans force ni vaillance », elle conquiert les femmes de familles guerrières et vaut aux Troyens la ruine. Mais au combat, les dons d'Aphrodite ne servent à rien (54-5). L'apostrophe révélatrice par laquelle Hector invective son frère revient au Chant XIII (III.39 = XIII.769), quand il part en quête des preux pour monter à l'assaut : 


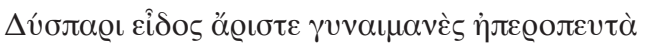

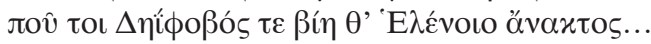

Ah! Pâris de malheur! premier pour la beauté, fou de femmes, séducteur ! où donc sont Déiphobe et la force de sire Hélénos? (XIII.769-70)

Le contraste est très marqué entre Pâris et les héros que recherche Hector: aux yeux de son frère aîné, il incarne tout le contraire de «la force de sire Hélénos » alors même qu'il le trouve au combat. Certes, Alexandre se défend. Mais tout au plus peut-il répondre que sa mère n'a pas enfanté en lui un homme «entièrement privé de vaillance» (776). Ce qui, dans sa bouche, n'est pas une litote. Il vient de reconnaître qu'en d'autres occasions il s'écarte davantage du combat. L'euphémisme réside plutôt là.

Certains tiennent qu'Hector est injuste ici et Pâris poli dans sa réponse ${ }^{66}$ : après tout, pour l'heure, il est bien au front! Ce n'est évidemment pas faux. Mais il me semble que le véritable sens de cette scène est ailleurs. À la différence d'un Achille, d'un Diomède ou encore d'Hector luimême, Pâris insulté ne réagit jamais avec indignation ni véhémence ${ }^{67}$. $\mathrm{Au}$ Chant III, il donnait raison à son frère (59-75); un peu plus tard, il éludera les sarcasmes d'Hélène (438-46); au Chant VI (333-41), quand Hector croit qu'il boude après sa défaite contre Ménélas ${ }^{68}$, il acquiesce à nouveau; ici, sa défense est conciliante. Cependant, cette attitude ne tient pas seulement au charme aimable du personnage, mais au fait qu'il est profondément éhonté: il ignore tout de la vergogne, vé $\mu \varepsilon \sigma \iota \varsigma$, cette honte mêlée de colère que l'on éprouve face au regard désapprobateur d'autrui (335), comme de la pudeur, aió́s, le sentiment intime de l'honneur, la « conscience ». Hélène, confrontée aux malheurs qu'elle a causés autour d'elle, le déplore (cf. 350-1). Ménélas avait vu juste: au lieu d'être ferme

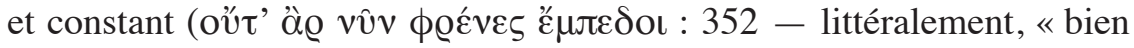

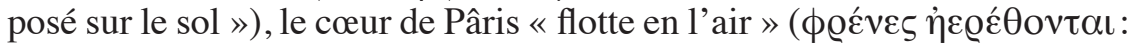
III.108). Le Priamide est insensible au sentiment de l'honneur. Or c'est pourtant cette aỉó́s qui prévient la fuite, qui permet de remporter la

66. Cf. JANKo, The Iliad cité, p. 141-2.

67. Pour Achille, cf. I.1; pour Diomède, IX.31-49; pour Hector, XVII.170-82.

68. À entendre Hector critiquer la «colère » (VI.326) de Pâris, nous pourrions croire qu'il le prend pour un nouvel Achille (cf. XVI.30-1 et 60-1)! Mais son frère est bien incapable d'une telle indignation (VI.335). Sur ce point, cf. Lindalu Coluins, «The Wrath of Paris: Ethical Vocabulary and Ethical Type in the Iliad», The American Journal of Philology 108, n² 2, 1987, p. 220-32. 
gloire, et le secours que seule procure la vaillance, l'ó $\lambda x \eta^{69}$. Pâris n'est pas seulement le favori d'Aphrodite. Son absence de pudeur l'entraîne

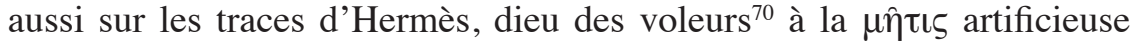

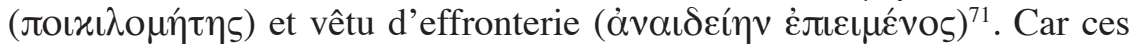
deux divinités partagent de nombreux traits, en particulier leur goût pour la fourbe et la séduction.

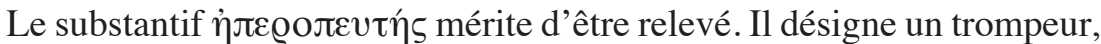

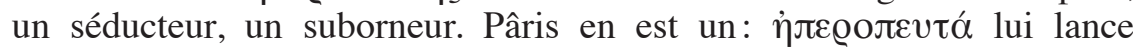
régulièrement Hector, dernier terme de l'apostrophe formulaire et résumé

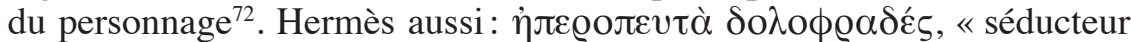
qui n'a que la ruse en tête! ${ }^{73}$ l'interpelle son frère Apollon. Quant à Aphrodite, au Chant $\mathrm{V}$ de l'Iliade, le puissant Diomède condamne chez elle ce même défaut :

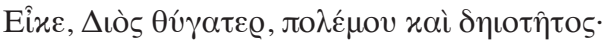

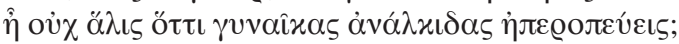

Quitte, fille de Zeus, la guerre et le carnage !

Ne suffit-il pas que tu séduises des femmes sans vaillance? (V.348-9)

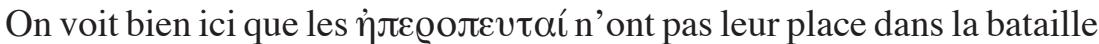
(cf. 330-3). Ils sont terrifiés par la guerre (350-1). Leurs talents sont tout opposés à ceux d'un preux. Cypris ne se fie ni à la vigueur, ni à la valeur, mais à la fourbe ${ }^{74}$. Elle ne cesse de suborner Hélène (III.399). Elle respire la ruse (III.405) et met ses armes aux services de ses favoris (III.402): Pâris le « séducteur »n'en connaît pas d'autres. À eux deux, ils rendent la beauté même suspecte ${ }^{75}$.

Le Priamide se comporte à la guerre non comme un héros hors des lignes, mais comme un « chasseur noir ». Or cette chasse rusée est aussi celle qu'il emploie pour séduire les belles. Diomède dénonce en lui un « lorgneur de

69. Les rois, les dieux aussi, aiment le rappeler aux combattants. Ainsi Agamemnon au Chant V (529-532). Plus loin, c'est Héra qui tance les Achéens (787 sq.) en opposant expressément la beauté des apparences à la véritable valeur, incarnée par Achille. Voir

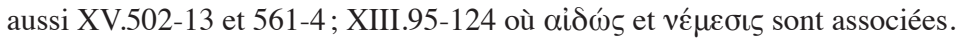

70. Cf. Hymne homérique à Hermès, $14 ; 175 ; 214 ; 292 ; 336 ; 340$ etc.

71. Hymne homérique à Hermès, 155-6.

72. Cf. KIRK, op. cit., p. 271.

73. Hymne homérique à Hermès, 282. Voir aussi 577-8.

74. Sur la $\mu$ îtı丂 d'Aphrodite, cf. Detienne et Vernant, op. cit., p. 269 sq.

75. Au Chant XVII, Glaukos, furieux que les Troyens ne mettent pas plus d'ardeur à dépouiller le cadavre de Patrocle, tance Hector en opposant sa beauté à ses talents de combattant (142). 


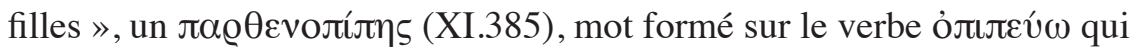
signifie «épier, guetter » et que l'épopée associe avec ceux qui frappent de loin, par surprise, sans être vus. Quand Hector s'apprête à affronter Ajax en duel au Chant VII, il renonce expressément à ce type d'attaque (234-43). Le Priamide est «en $\mu$ îtıৎ l'égal de Zeus » (47), à l'instar du seul Ulysse. Toute sa réplique souligne son expérience, sa science de la guerre (cf. l'anaphore du verbe «savoir »: 236, 237, 238, 240, 241). Pourtant, à la différence de Pâris chez qui Diomède ne découvrira qu'une femme, qu'un gamin insensé ${ }^{76}$, Hector est tout le contraire: « ne me tâte pas comme un faible enfant, ou comme une femme, qui ne connaît pas le labeur guerrier! », lance-t-il ainsi d'entrée à son adversaire (235-6). Comme Ajax l'expliquait tantôt à ses compagnons, dans ce duel, Hector pourrait ne pas recourir à la force (ßín: 197), mais porter un coup fondé

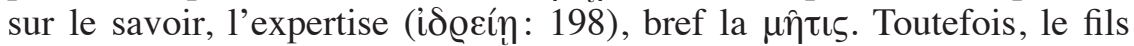
de Priam refuse ici de mettre ses idées au service de la ruse. Il ne veut pas frapper «sans être vu », «après avoir épié » sa proie précisément

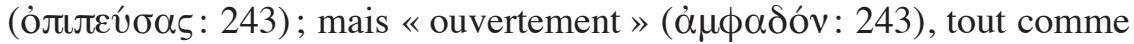
Ajax lui-même (cf. $\alpha \mu \phi \alpha \delta i ́ \eta v: ~ 196)^{77}$.

Pâris, lui, emprunte la voie inverse. Il ne se montre à Diomède qu'une fois son adversaire cloué au sol par son trait rusé (XI.376-9). On se souvient d'Euphorbe, fierté de la jeunesse troyenne (XVI.808), noté pour sa belle chevelure (XVII.51-2), qui se formait encore il y a peu à la guerre (XVI.811). Au Chant XVI, il frappe Patrocle à distance, « par-derrière, dans le dos » (806-7). Il ne s'est approché qu'à la faveur du vertige qui s'est emparé du héros quand Apollon l'a assommé, là encore «par-derrière » (790-1). Mais dès que Patrocle se retourne, tout désarmé qu'il soit (815), Euphorbe fuit et se mêle à la foule (813-4). Affronter Patrocle en face, entrer dans un duel avec Ménélas quelques vers plus loin, c'est pour le jeune homme une épreuve dans laquelle faire l'essai « ou de la vaillance ou de la fuite» (XVII.41-2). Contre le fils de Ménœtios, une fois son coup donné à la dérobée, il a choisi la fuite. Face à Ménélas au contraire, il tente sa chance et perd la vie. C'est là très précisément le parcours de Pâris au Chant III, chasseur d'abord, armé de forfanterie, fuyard ensuite, en archer qui ne sait pas tenir de pied ferme, duelliste enfin, vaincu par l'Atride. Hélène, du reste, ne se prive pas de moquer les fanfaronnades de Pâris et de souligner combien Ménélas est «plus fort » que lui (III.428-36). Dans l'épreuve de

76. Cf. XI.389.

77. Sur l'attitude d'Ajax, pour qui « le meilleur dessein (vóo $)$, la meilleure idée ( $\mu \eta ̂ \tau ı \varsigma)$, c'est de mettre au contact, au corps à corps, les bras et la fougue », cf. XV.509-10. 
force, celle où il faut l'emporter par la vigueur des bras et de la lance ( $\sigma \eta \hat{~} \tau \varepsilon$

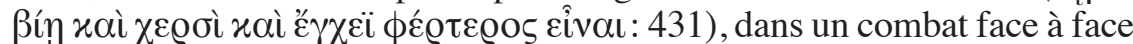

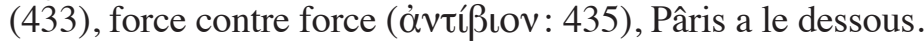

Si au Chant III, dans le discours de Ménélas, Priam, face à ses fils, incarne la force, la ßín, c’est d'abord parce que Pâris symbolise lui la fourbe, la fraude, la déloyauté, bref la $\mu$ $\imath ı \varsigma$ scélérate. Dans cette opposition, les bonnes idées, celles qui s'avèrent « les meilleures » (110), sont rapportées à la force, bien qu'imaginées par un vieillard, parce qu'elles sont franches et claires. La bonne $\mu \hat{\eta} \tau \iota \varsigma$ est vigoureuse. Au rebours, la force que l'on croit déceler dans la jeune et éclatante beauté d'Alexandre n'est qu'illusion. Nous avons dit que Pâris était resté trop gamin pour connaître la vaillance ardente. Il est également trop puéril pour avoir de bonnes idées. De fait, Ménélas semble destiné dans l'Iliade à opposer sa valeur à la méchante

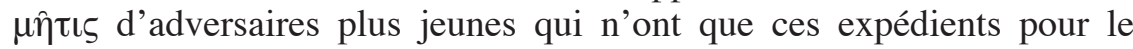
vaincre. Marcel Detienne et Jean-Pierre Vernant ${ }^{78}$ ont noté le parallèle entre cette scène où il affronte Pâris et un épisode du Chant XXIII, la course de char en l'honneur des funérailles de Patrocle. Il est éclairant.

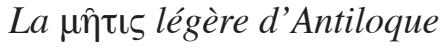

L'attelage d'Antiloque est moins bon que les autres. Son père Nestor,

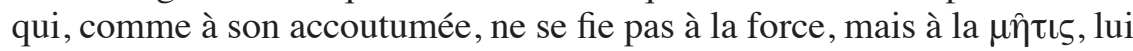
explique que sa seule chance de l'emporter est d'avoir des idées (XXIII.311 $s q$.). Au cocher qui se fie aux qualités physiques de son attelage, à sa ßín, Nestor préfère «celui qui, bien que menant des chevaux moins bons, connaît tous les tours », les $x \dot{c} @ \delta \varepsilon \alpha$ (322). Opportuniste, l'oubli n'a pas

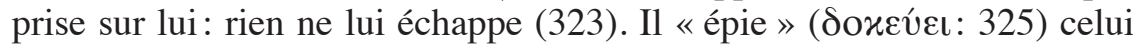
qui le devance et conduit sa course sans faillir, quand son adversaire, sûr

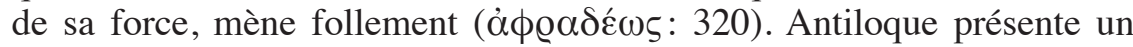
défaut: il est jeune (306). Nous retrouvons là l'opposition traditionnelle entre jeunesse et vigueur d'une part, vieillesse et sagesse de l'autre. Mais, outre qu'il est le fils de Nestor, il a eu les meilleurs professeurs, Zeus et Poséidon (307). La $\mu$ $ү \imath \iota \varsigma$ est d'abord affaire de science, d'expertise, en particulier quand il s'agit de conduire des chevaux, discipline aux

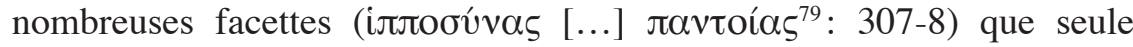

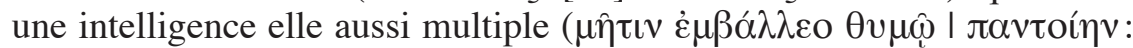
313-4) peut maîtriser. Antiloque possède mieux ce savoir que ses rivaux

78. Cf. Detienne et Vernant, op. cit., p. 23.

79. Sur les liens entre cet adjectif et la $\mu \hat{\imath} \tau ı$, Detienne et Vernant, op. cit., en particulier p. 19,25 et 32 . 
(309-11). Ainsi donc, pour Nestor, pas de doute, la victoire ira à la $\mu \eta \hat{\imath \iota \varsigma}$ et non à la ßín (315-8).

En outre, Antiloque a déjà fait la preuve, lors de ses combats passés contre les Troyens, de sa $\mu \hat{\imath} \tau \iota \varsigma$. Il a su «épier» $(\delta o x \varepsilon v ́ \sigma \alpha \varsigma: \text { XIII.545 })^{80}$ Thoon, avant de saisir le bon moment pour le frapper. Toujours aux aguets,

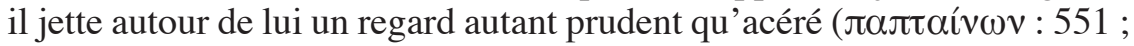
cf. XV.574) quand il mène à bien les plus grands exploits ${ }^{81}$.

Certes, Antiloque ne peut l'emporter sur Diomède: ce jour-là, Athéna donne la victoire au fils de Tydée (XXIII.405-6). Mais, grâce à ses « idées », il compte bien battre Ménélas. Et c'est là que tout se complique. L'Atride, bien que l'aîné dans ce duel, «l'emporte tant par la valeur que par la

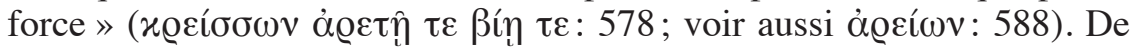
plus, ses chevaux sont moins âgés que ceux du fils de Nestor et, cette fois, la logique est respectée. Leur jeunesse est le gage de leur vigueur et ils se fatigueront moins vite que l'attelage d'Antiloque (cf. 444-5). Antiloque, lui, s'en remet à sa $\mu$ îtıร. Art de machiner, d'inventer, c'est grâce à elle, annonce-t-il à ses coursiers, qu'il va prendre le dessus (414-6). La ruse est

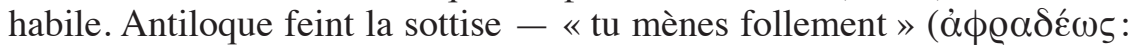
426), lui lance Ménélas - normalement réservée au cocher trop confiant dans la valeur de son attelage (319-20). Mais, en fait, grâce à ses idées, Antiloque confère à ses chevaux la force qui leur manquait jusque-là (4313). Le paradoxe est ici souligné: par la magie de la $\mu \hat{\imath} \tau \iota \varsigma$, le vieil attelage paraît retrouver la vigueur de sa jeunesse et son cocher, faible bien que jeune, la force de son âge. Car Antiloque connaît tous les «tours » que vantait son père (322):

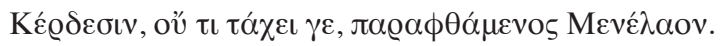

C'est grâce aux tours, et non à la vitesse, qu'il devance Ménélas. (XXIII.515)

La leçon de Nestor semble avoir porté ses fruits. Ménélas avait bien « des chevaux plus vites », mais il ne savait pas autant qu'Antiloque avoir des idées. En apparence, le jeune homme a su appliquer les conseils de son père et en profiter pour mieux figurer à l'arrivée ${ }^{82}$.

80. Cf. Detienne et Vernant, op. cit., p. 21-2.

81. Redisons-le: quand elle sert la bravoure, l'idée n'a rien de blâmable. C'est quand elle lui fait honte que les problèmes surgissent. Sur le double sens du verbe $\pi \alpha \pi \tau \alpha i v \omega$ (il note ensemble la prudence et l'agressivité) et son association au monde de la chasse ainsi qu'aux personnages d'Antiloque et de Teucros, cf. Steven H. LonsDaLe, «If Looks Could Kill : $\pi \alpha \tau \tau \alpha i ́ v \omega$ and the Interpenetration of Imagery and Narrative in Homer », The Classical Journal 84, n ${ }^{4}$, 1989, p. 325-33.

82. Sur le détail de cette course, cf. Michael Gagarin, «Antilochus'Strategy: The 
Cependant, le fils d'Atrée ne se laisse pas faire. S'emparant du fameux sceptre de ses pères, gage d'autorité et d'équité, il porte réclamation (56885). La ruse d'Antiloque n'aura pas raison de sa valeur. Une nouvelle fois, les termes de l'affrontement entre le jeune fils de Nestor et le vétéran qu'est Ménélas sont paradoxaux. Alors que la ruse est l'apanage de 1'expérience, c'est Antiloque qui en a usé en connaissance de cause (585), pour faire gagner ses chevaux, « bien plus mauvais » que ceux de l'Atride (572). En outre, la $\mu \hat{\imath} \tau \iota \varsigma$ dont il a fait preuve passe aux yeux de Ménélas pour de la sottise, et non de l'intelligence. Le fils de Nestor était « avisé naguère » ( $\varrho o ́ \sigma \theta \varepsilon v ~ \pi \varepsilon \pi v v \mu \varepsilon ́ v \varepsilon: 570$; voir aussi 440), mais non durant la course. Sa victoire fait honte à la valeur (571). Ménélas, de son côté, bien que plus âgé, « est personnellement plus puissant par la valeur comme par

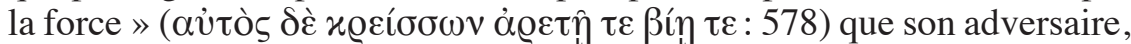
au point qu'il pourrait, grâce à ce statut, faire violence au fils de Nestor

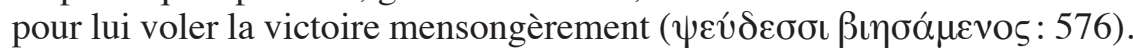
La ßín, à la fois vigueur et contrainte, appartient tout entière à l'Atride. Mais, à la différence d'Antiloque qui, pour Ménélas, fait un mauvais usage de ses idées, le fils d'Atrée en use droitement et veut rendre un jugement irréprochable (579-80). Son jeune adversaire, du reste, redevenu avisé ( $\pi \varepsilon \pi v v \mu \varepsilon ́ v o s$ : 586), se rend à ses raisons et dénonce la cause, toute traditionnelle, de son irréflexion, sa propre jeunesse :

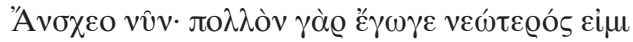

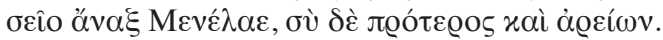

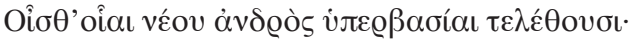

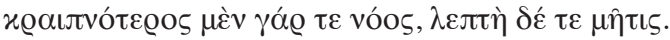

Sois patient à cette heure. Je suis pour ma part bien plus jeune que toi, sire Ménélas; et tu es plus âgé et meilleur.

Tu sais ce que sont les extravagances d'un jeune homme.

L'esprit en lui est trop vif et les idées minces. (XXIII.587-90)

Cette réponse fait nettement écho à la scène du Chant III où Ménélas en appelle à la « force de Priam » (П@ı́́ $\mu$ oıo ßínv) contre la déloyauté de ses fils. La ruse d'Antiloque est condamnable parce qu'elle est l'œuvre de la jeunesse. Le fils de Nestor est le cadet de Ménélas (XXIII.587-8). Ses excès

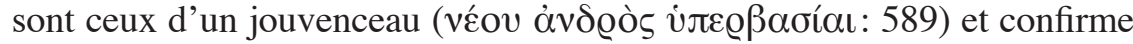
les craintes de l'Atride face aux Priamides ( $\mu$ í $\tau \iota \varsigma$ vं $\tau \varepsilon \varrho \beta \alpha \sigma i ́ n ~ x \tau \lambda$.:

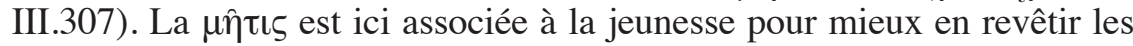
défauts: orgueil, puérilité, infidélité. Ménélas lui-même en est convaincu:

Chariot Race in Iliad 23 », Classical Philology 78, n 1, 1983, p. 35-39. 


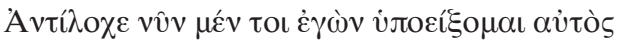

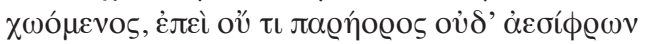

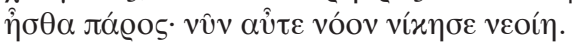

Antiloque, pour l'heure je dois moi-même te céder en dépit de ma colère: car étourdi ou insensé,

tu ne l'étais pas avant; mais aujourd'hui la jeunesse a vaincu la raison. (XXIII.603-4)

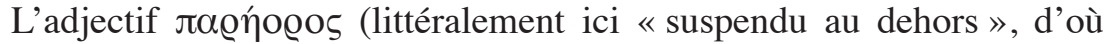

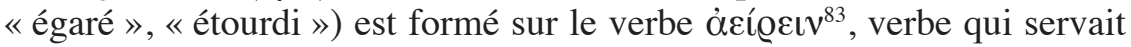
déjà à Ménélas pour décrire l'esprit «volage » des Priamides (III.108:

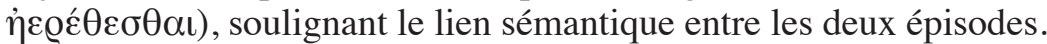

Finalement, c'est vraiment Antiloque qui menait son char «follement »

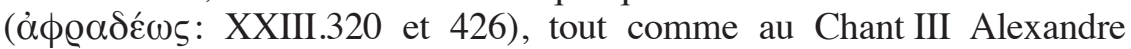
agissait «follement» en voulant affronter Ménélas en combat singulier

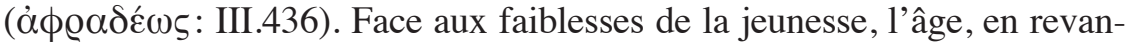
che, est tout entier placé du côté de la vigueur. Dans tout ce récit, comme dans les passages du Chant III auxquels il fait écho, la vigueur des vétérans est également synonyme de sagesse et de valeur. Priam, même vieux, se ramenait à sa force. Ménélas, quant à lui, est non seulement l'aîné d'Antiloque, il est aussi «meilleur» que lui (XXIII.588). Chez l'Atride, comme

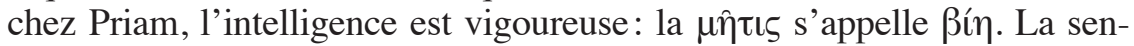
tence est claire que lui livre - sur un ton très personnel - le fils d'Atrée :

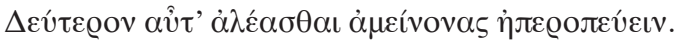

Évite une autre fois de chercher à jouer ceux qui valent mieux que toi. (XXIII.605)

Nous retrouvons ici les dons d'Aphrodite et d'Hermès. Antiloque s'est

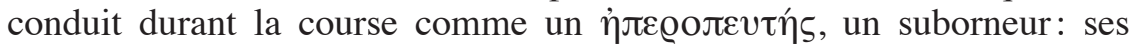

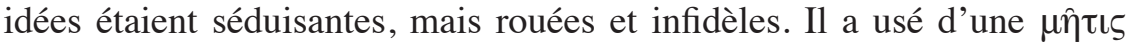

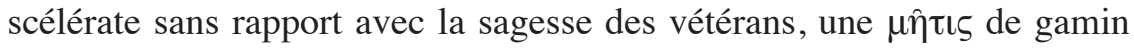
ou de femme, celle que condamne le puissant Diomède chez Aphrodite et Pâris. Bref, il a corrompu les idées de son père.

83. Chantraine, op. cit., s.v. àzí@ $\omega, 1$ et 2, p. 22-3. Voir aussi Nicholas Richardson, The Iliad: A Commentary Vol. VI, Books 21-24, Cambridge, 1993, p. 231, ad. loc. Au départ,

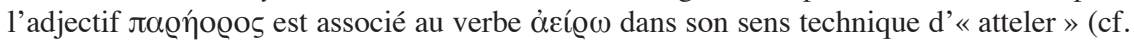
$\alpha \dot{\varepsilon} \varrho \omega 2$ ) et désigne un cheval de volée « qui est attaché à part » (le préverbe $\pi \alpha \varrho \alpha$-s'oppose à $\sigma u v$-). Or la nature du cheval de volée dans l'Iliade est précisément de mettre en péril le héros et son attelage, au point que Geoffrey Kirk le qualifie de « reckless », téméraire, imprudent, irréfléchi: cf. KIRK, The Iliad: A Commentary Vol.II, Books 5-8, Cambridge, 1990, p. 255-6 (VII.155-6) et 304-5 (VIII.78-91: sur ce passage cf. supra, «Vieillesse ennemie »). Sans doute n'est-il pas indifférent qu'un cheval de volée soit à l'origine de la mort d'Antiloque lui-même dans l'Éthiopide (cf. Pindare, VI Pythique, 28-42). 
Cette leçon est confirmée dans les vers qui suivent à travers trois scènes révélatrices. D'abord, Achille offre le cinquième prix au vieux Nestor justement (XXIII.615-52). Bien que trop vieux pour concourir (643-4), il n'en mérite pas moins d'être honoré parmi les Achéens pour ses vertus passées et présentes (648-9). La faute de son fils ne rejaillit nullement sur sa propre $\mu \eta \hat{\imath \iota \varsigma, ~ q u i ~ r e s t e ~ a ̀ ~ l ' h o n n e u r . ~ C a r ~ l e s ~ i d e ́ e s ~ d u ~ v i e i l ~ h o m m e ~}$ sont denses, solidement ajustées (

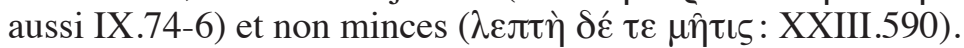

Ensuite, lors de l'épreuve de lutte (XXIII.708 sq.) qui affronte « le grand Ajax », tenant de la force brute, comme nous l'avait révélé son duel contre

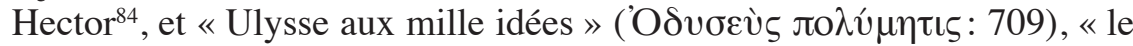

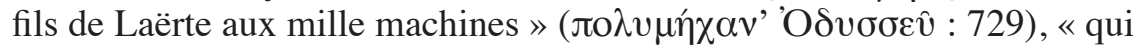

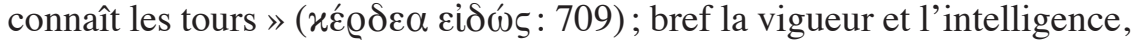

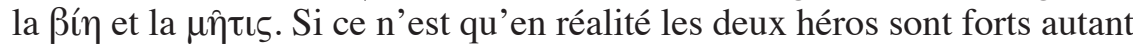
qu'experts: face au grand Ajax « Sa Force Ulysse puissamment résiste » (720). Ni l'un ni l'autre ne peut prendre le dessus grâce à la seule vigueur.

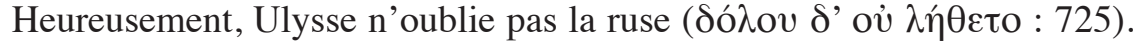
Il arrive à renverser Ajax, mais sans parvenir à le vaincre (726-32). Le fils

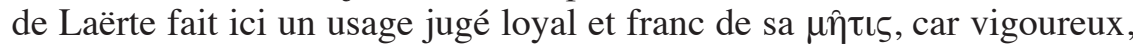
appuyé sur la force du combattant. Si les ruses d'Ulysse sont multiples et

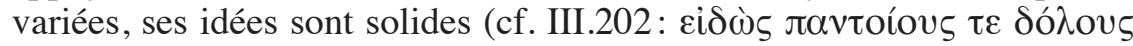

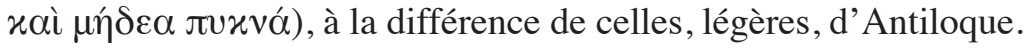

Enfin, quand a lieu le concours de vitesse (XXIII.740 sq.), trois héros entrent en lice : 1'autre Ajax, le rapide fils d'Oilée (754), « Ulysse aux mille idées » (755) et Antiloque en personne. Le vif Ajax part naturellement en tête, suivi d'Ulysse qui le talonne (758-7). Le fils de Laërte fait alors appel à l'aide d'Athéna, qui fait trébucher le fils d'Oilée (774) et lui donne la victoire $^{85}$. Et Antiloque dans tout cela? Il avait tous les atouts pour lui : jeunesse et vitesse ${ }^{86}$. Antiloque « l'emportait à la course sur tous les jeunes gens » (756). Et pourtant, une fois encore, il est battu par plus vieux que lui

84. Cf. supra, «Les dons charmants d'Aphrodite»; voir aussi XV.510.

85. Lors de la course de char, c'est Athéna déjà qui avait fait triompher Diomède en ruinant les espoirs du meilleur concurrent de l'épreuve, Eumèle, « qui excelle dans l'art de mener les chevaux» (XXIII.289). Comme le remarque Achille en cette occasion: «le

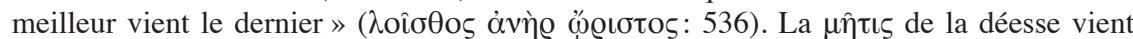
troubler l'ordre naturel fondé sur la valeur, la force des adversaires. Mais nul ne songe à lui en faire grief: la force et l'idée sont inséparables; cela fait partie du jeu. Achille lui-même, qui honore le vieux Nestor ici, puis Priam au Chant XXIV, semble l'avoir compris: la ßín seule ne peut pas tout.

86. Sur les qualités d'Antiloque, voir aussi l'Odyssée : 3.111-2 et 4.201-2. 
(787-92). Dans ces jeux, la jeunesse n'est pas de taille face aux vétérans, ni dans les épreuves de force, ni dans les joutes où compte la science. Antiloque est trop jeune pour être assez fort. Trop jeune aussi pour faire bon usage de ses idées ou de celles de son père. Ménélas au rebours, Nestor aussi, voire Ulysse, incarnent à la fois une force vigoureuse et franche et un savoir employé à bon escient. L'Atride en particulier sort grandi de ses déboires avec Antiloque : il apparaît sous les traits d'un bon roi, rendant de

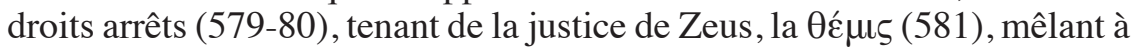
une supériorité toute martiale, fondée sur la force et la valeur et rejetant la violence et le mensonge (573-8), les bonnes idées et le talent oratoire d'un Priam ou d'un Nestor. Finalement seule l'alliance de la ßín et de la $\mu \eta \hat{\tau ı \varsigma ~}$ que symbolise précisément parfaitement la formule «Sa Force Priam » et qu'incarne Ménélas lui-même à cette heure assure le succès.

Pour surprenante qu'elle est, l'expression П@ıó $\mu$ oı ßín paraît désormais tout à fait pertinente. La «force» peut effectivement se rassembler en Priam, tout vieux qu'il est. Parce qu'elle est équitable, franche, honnête, sa

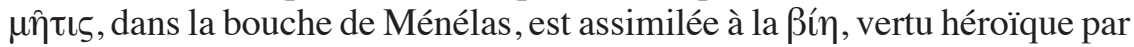
excellence. Au rebours, la jeunesse de son fils Alexandre, celle d'Antiloque, gage habituellement de qualités physiques et martiales, devient ici

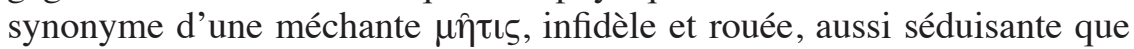
ruineuse. On voit bien comment ces associations obéissent à des « thèmes » qui parcourent l'épopée. Vieillesse, intelligence, droiture forment avec leurs antonymes, jeunesse, extravagance, déloyauté, des combinaisons dans lesquelles les termes de cette opposition «traditionnelle», voire « formulaire », ne sont pas figés, mais glissent d'un personnage à l'autre en fonction des circonstances ${ }^{87}$. Au Chant XV, Antiloque était aux yeux de Ménélas jeune, vif et vaillant. Au Chant XXIII, s'il reste tout jeune, il se montre lent désormais et fait insulte à la valeur. Entre les deux, le fils de Nestor, d'un allié qu'il était pour Ménélas, est devenu son rival pour le deuxième prix à la course de char, une cavale - nouvelle Hélène - et a pris les traits de l'ennemi de toujours: Pâris.

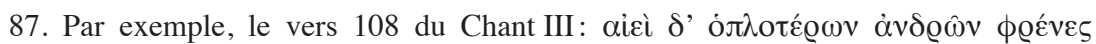

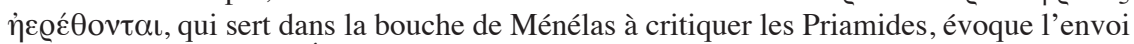

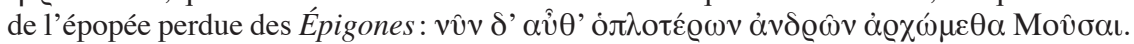
Or, quand on songe que, dans l'effectif achéen, nombre de héros faisaient justement partie de ces « hommes mieux armés par la jeunesse » qui emportèrent Thèbes, que ces Épigones insistent dans l'Iliade, Diomède en tête, sur le fait qu'ils valent, eux, tant par la force que par l'idée, mieux que leurs pères (cf. IV.405), victimes justement de leur excès, le contraste avec les Priamides n'en est que plus criant et la critique de ces derniers que plus mordante, eux qui laissent non seulement la bonne $\mu \hat{\eta} \tau \iota \varsigma$, mais même la ßín, à leur vieux père. 
Chez Priam, au contraire, la force s'enracine. Faible, mais sage au Chant III, père d'Alexandre, mais honnête et franc, « au seuil maudit de la vieillesse ${ }^{88}$, mais fort de ses idées, il ne se renie nullement quand arrive le terme de l'épopée. Lors de son entrevue célèbre avec Achille, à qui il est venu réclamer le corps de son fils, Hector, il force l'admiration du meilleur des Achéens, ßí incarnée, meurtrier des siens. La supplique de Priam, dont nous avons noté les talents « musicaux », l'émeut aux larmes (XXIV.507 sq.). Le Péléide est stupéfait par le courage et l'audace du vieil homme qui s'est rendu seul au camp des Achéens (518-21). Certes, Achille finit par lui rappeler qu'il ne doit cet exploit qu'à l'aide d'un dieu. Nous savons que c'est Hermès, sur l'ordre de Zeus, qui l'a guidé (153-4 et 460-7), car nul Troyen, même en pleine jeunesse, n'oserait s'aventurer près des nefs (565-6). Mais le souverain troyen n'en a pas moins agi en héros, tout âgé qu'il fût (362-9), prêt à mourir pour rapatrier la dépouille de son fils (224-7). Quant à l'astuce d'Hermès, elle est pour l'heure au service de cet héroïsme et non des ruses et des rapines d'un Pâris. Priam, du reste, dénonçait juste avant de partir ses fils survivants, Alexandre en tête (cf. 249), qui ne sont que des menteurs et des danseurs (259-62). Face à la frivolité lâche et déloyale des Priamides, l'idée de leur père, son vóos

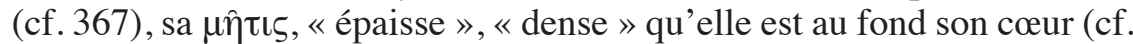

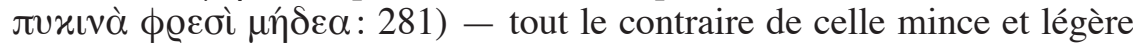
d'un jouvenceau - témoigne bien de sa force. Priam mérite d'être appelé

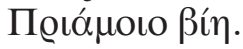

La composition de l'aède apparaît donc finalement comme autant de vertigineux entrelacs dont les formules et, à travers elles, les « thèmes épiques » qu'elles évoquent et convoquent, forment la matière première.

\section{Le cour « hyperviolent » du vieux Nestor}

Pour conclure cette étude, je voudrais expliquer par une lecture comparable une autre étrange formule, très proche de «Sa Force Priam », tant sur le fond que pour la forme, mais qui se trouve au Chant 15 de l'Odyssée cette fois.

Au terme de son séjour à Sparte, Télémaque est pressé de rentrer à Ithaque : Athéna 1'y a expressément engagé (1-47). Accompagné de son guide, Pisistrate, fils de Nestor, il regagne Pylos où l'attend son navire. Toutefois, pour ne pas perdre un moment, Télémaque ne veut pas faire halte chez Nestor: il doit partir au plus vite et craint que le vieillard ne cherche à le retenir (198-200). Pisistrate accède à sa demande, mais le met en garde

88. Cf. XXIV.486-7. 
contre la colère de son père (209-14). Or la formule qu'il emploie est très surprenante. Il sait combien le cœur de Nestor - entendons le siège de sa colère (cf. 214) - est fort, ou plutôt ici «violent », à l'extrême ( $\theta v \mu$ òs vंлદ́@ßı૬: 212). Si nous appliquons les catégories traditionnelles à ce passage, le fils de Nélée, un vieillard, ne devrait pas être associé à une telle épithète. Elle note la vigueur, la force, la ßín dans tous ses excès. Bref, les extravagances de la jeunesse. Dans l'Odyssée, ce sont les prétendants, émules de Pâris ou d'Euphorbe, aussi gamins que roués, qui font preuve d'une arrogance et d'une cupidité par trop violente ( $\mu \nu \eta \sigma \tau \eta ̂ \varrho \varepsilon \varsigma ~ v ं \pi \varepsilon ́ \varrho \beta เ o v$

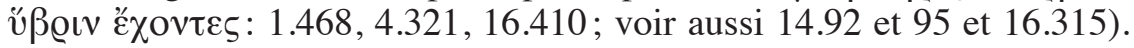
Tout le contraire, donc, du vieux Nestor.

Mais ce n'est pas tout. Le texte se brouille encore davantage si nous songeons que nous avons déjà croisé cette formule, à l'identique, dans l'Iliade. Au Chant XVIII, en effet, le troisième fils de Panthoos, « Polydamas l'avisé » ( $\pi \varepsilon \pi v v \mu \varepsilon ́ v o \varsigma$ : 249) qui, à la différence d'Euphorbe et d'Hypérénor, brille au conseil (252), mais aussi au combat (cf. XIV.40932 ), en use pour expliquer la crainte que lui inspire le « cœur violent à

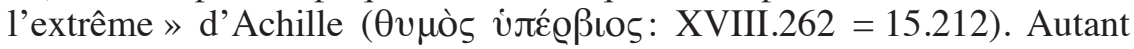
dire que l'écho ainsi produit est cocasse. Associer ainsi Nestor, le vieillard

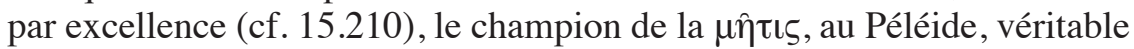
force de la nature, produit un véritable oxymore.

En dehors d'un pur hasard formulaire, s'agissant de l'Odyssée, nous sommes en droit de soupçonner une parodie ${ }^{89}$. Cette épopée n'est possible qu'après l'Iliade et entretient avec son aînée des rapports compliqués. Pour reprendre les termes de François Hartog, «l'Odyssée est en position d' "histoire" ${ }^{90}$. Le point de vue est différent, aussi inversé en fait que le sont les figures mêmes d'Achille et d'Ulysse. Le Péléide acquiert une gloire immortelle en renonçant à une vieillesse obscure dont il apprécie pourtant l'attrait (IX.401-16) pour embrasser une mort héroïque qui le fauche en pleine jeunesse (XVIII.98-121). Au contraire, l'Odyssée, elle, assure le renom de son héros parce qu'il survit. Ce n'est pas la mort, mais son retour qui entérine la gloire d'Ulysse ${ }^{91}$. Tant qu'on le croit mort, mais

89. David Monro, dans son commentaire de l'Odyssée, note à propos de ce jeu d'écho que le poète n'était certainement qu'à demi sérieux : Homer's Odyssey Books XIII-XXIV, Oxford, 1901, p. 54.

90. François Hartog, «Premières figures de l'historien en Grèce: historicité et histoire », dans Nicole Loraux et Carles Miralles (éd.), Figures de l'intellectuel en Grèce ancienne, Paris, p. 127.

91. Cf. NAGY, Le meilleur des Achéens cité, p. XII-XIII de la préface à l'édition de 1999 et p. 35 sq. Il n'est pas certain cependant que l'Odyssée parvienne à ses fins. Pour Pietro 
sans savoir ni où ni comment, sans pouvoir le célébrer, son $x \lambda \hat{\varepsilon}$ o 5 est en perdition ${ }^{92}$. Les propos que lui tient Achille lors de sa « katabase » ne font qu'affirmer ce changement de perspective (11.482-91). Dans le dialogue constant qu'entretiennent les deux épopées, c'est toujours l'Iliade qui donne le ton ${ }^{93}$. Aussi, appliquer à Nestor une formule qui appartient au fils de Pélée pourrait n'être qu'un pied de nez de l'Odyssée à l'Iliade,

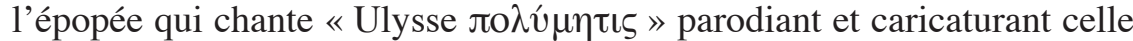

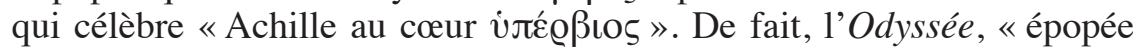
nostalgique, celle d'un retour impossible et désiré vers l'épopée, vers 1'Iliade $\gg{ }^{94}$, mime des affrontements iliadiques. Le meurtre d'Antinoos et de ses sbires donnera lieu à des jeux d'écho, relevés en détail par Pietro Pucci $^{95}$, qui conféreront au fils de Laërte les mêmes vertus héroïques que celles déployées par le Péléide lors de son aristie, de ce carnage immense qu'il fait des Troyens au Chant XX et suivants de l'Iliade.

Pourtant, il est une autre explication que l'on peut avancer pour éclairer cette curieuse mention du « cœur hyperviolent » de Nestor. Télémaque et Pisistrate sont tout jeunes (cf. 3.357-79). Le fils de Nélée est lui très vieux. Il était déjà âgé au temps de la guerre de Troie et dix ans ont passé. Or, dans la scène qui nous intéresse, ce sont les deux jeunes gens qui cherchent à jouer le vieux meneur de char. Leur plan n'est pas décrit expressément comme une « idée », mais Télémaque tient de son père et n'a rien à envier à sa $\mu \hat{\imath} \tau \iota \varsigma$, si l'on en croit Athéna (cf. 2.278-9). Il est toujours «avisé » $(\pi \varepsilon \pi v v \mu \varepsilon ́ v o \varsigma)^{96}$, cette qualité même dont jouissait naguère Antiloque avant de faire honte à Ménélas (cf. XXIII.440 et 570). En outre, sa précipitation lui est inspirée par Athéna en personne et il s'agit bien de se soustraire aux

Pucci, Ulysse n'obtient de gloire dans l'Odyssée que pour ses exploits sous les murs de Troie. Le fait même qu'il rentre chez lui «lui ôte tout $x \lambda \varepsilon$ ó polutropos cité, p. 207-8. Quant au $x \lambda \varepsilon ́$ os proprement odysséen, c'est peut-être Pénélope plutôt qu'Ulysse qui en est la clef: cf. NAGy, Le meilleur des Achéens cité, p. 38 ; PuccI, Ulysse polutropos cité, p. 298 et p. 301, note 12.

92. Philippe Rousseau, «Instruire Persès: Notes sur l'ouverture des Travaux d'Hésiode », dans Fabienne Blaise, Pierre Judet de la Combe et Philippe Rousseau (éd.), Le métier du mythe : lectures d'Hésiode, Lille, 1996, p. 101, en particulier la note 26.

93. Cf. Rousseau, préface à P. Pucci, Ulysse polutropos cité, p. 19-20.

94. Hartog, op. cit., p. 136.

95. Cf. Pucci, Ulysse polutropos cité, p. 183-91. Les passages concernés sont: Iliade, XXI.18-24 et 71-4 et Odyssée, 22.297-312. On peut faire une analyse comparable à propos de l'affrontement final entre les parents des prétendants massacrés et Ulysse, accompagné de Télémaque et Laërte (24.465 sq.).

96. Les occurrences de cette épithète appliquée à Télémaque abondent: $1.213 ; 1.230$; $1.306 ; 1.345$ etc. 
attentions du vieillard qui sont présentées comme autant de contraintes (cf. 15.200 et 212-4). Autrement dit, thématiquement, et donc formulairement, l'idée est associée dans cet épisode précis aux deux jeunes princes tandis que, par le jeu des oppositions traditionnelles, la force revient à celui qui se dresse sur leur route et se trouve être un vieillard. Télémaque faisant figure de héros, son projet, qui a l'aval du Ciel, est paré de dehors flatteurs, tandis que les obstacles qu'il affronte se teintent de couleurs sombres. Sa

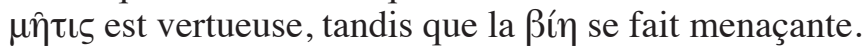

Finalement, peut-être Nestor n'est-il violent que de l'astuce de ses jeunes adversaires, tout comme Priam n'était fort que de la fourbe de ses fils. 